\title{
Maximizing the performance of pump inducers using CFD-based multi-objective optimization
}

\author{
Trupen Parikh $^{2} \cdot$ Michael Mansour ${ }^{1,2}\left[\right.$ Dominique Thévenin ${ }^{2}$
}

Received: 3 May 2021 / Revised: 23 August 2021 / Accepted: 29 September 2021 / Published online: 18 December 2021

(c) The Author(s) 2021

\begin{abstract}
Pump inducers are usually employed within a limited flow rate range since the performance is known to drop out significantly far from their design point. Therefore, finding an optimal geometry that ensures efficient operation for a relatively wide range of flow rates is challenging. The present study tackles this problem using multi-objective optimization to identify optimal inducer configurations, delivering high performance for a wide flow range. 3D RANS single-phase turbulent simulations were performed using the $k-\omega$ turbulence model. The optimization was done by employing the Non-dominated Sorting Genetic Algorithm (NSGA-II) coupled with computational fluid dynamics (CFD). An established in-house flow optimization library (OPAL++) was used to automatically control the numerical simulations. The objective is to optimize the inducer geometrical parameters to simultaneously maximize the efficiency and pressure head curves, considering different flow rates, i.e., $80 \%$ (part-load), $100 \%$ (nominal), and 150\% (overload) of the optimal flow rate for the considered pump. The optimization involves 8 most relevant design parameters, i.e., the axial blade length, blade sweep angle, blade pitch, hub taper angle, tip clearance gap, blade thickness at the hub, blade thickness at the tip, and the number of blades. A total of 5178 simulations over 37 generations have been needed to get a Pareto front containing 5 optimal configurations. This article discusses quantitatively the influence of each geometrical parameter on flow behavior and inducer performance. The results reveal in general that blade length, blade sweep angle, tip clearance gap, and blade thickness should be kept low for the considered application; inducers with high hub taper angles and 3 blades lead to optimal performance.
\end{abstract}

Keywords Multi-objective parameter optimization · Pareto front · Pump inducer · Efficiency · Pressure head · Computational fluid dynamics (CFD)

\section{Nomenclature}

Roman symbols

A Pipe cross-sectional area $\left[\mathrm{m}^{2}\right]$

$A_{1} \quad$ Pipe cross-sectional area at pressure sensor 1 $\left[\mathrm{m}^{2}\right]$

$A_{2} \quad$ Pipe cross-sectional area at pressure sensor 2 $\left[\mathrm{m}^{2}\right]$

$A_{\Delta p} \quad$ Area under the pressure head curve $[\mathrm{Pa}]$

Responsible Editor: Emilio Carlos Nelli Silva

Michael Mansour

michael.mansour@ovgu.de

1 Mechanical Power Engineering Department, Faculty of Engineering - Mataria, Helwan University, Cairo 11718, Egypt

2 Laboratory of Fluid Dynamics \& Technical Flows, University of Magdeburg "Otto von Guericke”, 39106 Magdeburg, Germany
$A_{\triangle P \max } \quad$ Maximum area under the pressure head curve $[\mathrm{Pa}]$

$A_{\eta} \quad$ Area under the efficiency curve

$F_{\mathrm{P}} \quad$ Pressure force [N]

$F_{\mathrm{S}} \quad$ Shear force [N]

$p_{1} \quad$ Static pressure at sensor $1[\mathrm{~Pa}]$

$p_{2} \quad$ Static pressure at sensor $2[\mathrm{~Pa}]$

$P \quad$ Blade pitch [m]

$p \quad$ Pressure [Pa]

$D_{\mathrm{t}} \quad$ Tip blade diameter [m]

$D_{\mathrm{h}} \quad$ Mean hub blade diameter [m]

$D_{\mathrm{m}} \quad$ Mean blade diameter [m]

$d \quad$ Suction pipe diameter [m]

$g \quad$ gravitational acceleration $\left[\mathrm{m} / \mathrm{s}^{2}\right]$

$L \quad$ Axial blade length [m]

$Q \quad$ Inlet flow rate $\left[\mathrm{m}^{3} / \mathrm{s}\right]$

$Q_{\text {opt }} \quad$ Optimal flow rate of the pump $\left[\mathrm{m}^{3} / \mathrm{s}\right]$

$N \quad$ Number of blades

$C \quad$ Tip clearance gap [m] 


$\begin{array}{ll}T_{\mathrm{t}} & \text { Tip blade thickness [m] } \\ T_{\mathrm{h}} & \text { Hub blade thickness [m] } \\ C_{\mathrm{m}} & \text { Mean blade chord length [m] } \\ n & \text { Rotational speed [rpm] } \\ n_{\mathrm{d}} & \text { Number of design parameters } \\ P_{\mathrm{f}} & \text { Fluid power [W] } \\ P_{\mathrm{Sh}} & \text { Shaft power [W] } \\ r & \text { Position relative to the torque axis [m] } \\ s_{\mathrm{m}} & \text { Mean azimuthal blade spacing [m] } \\ \mathbf{V} & \text { Fluid velocity vector [m/s] } \\ V_{1} & \text { Fluid velocity at pressure sensor } 1[\mathrm{~m} / \mathrm{s}] \\ V_{2} & \text { Fluid velocity at pressure sensor } 2[\mathrm{~m} / \mathrm{s}] \\ y^{+} & \text {Non-dimensional wall distance } \\ z_{1} & \text { Elevation at pressure sensor } 1[\mathrm{~m}] \\ z_{2} & \text { Elevation at pressure sensor } 2[\mathrm{~m}]\end{array}$

\section{Greek symbols}

\section{$\beta \quad$ Hub taper angle $\left[{ }^{\circ}\right]$}

$\theta \quad$ Blade helix angle $\left[{ }^{\circ}\right]$

$\Delta P \quad$ Inducer pressure head [Pa]

$\Delta P_{\text {maz }} \quad$ Maximum inducer pressure head [Pa]

$\Delta P_{\text {opt }} \quad$ Inducer pressure head at optimal flow [Pa]

$\Delta P_{\text {over }} \quad$ Inducer pressure head at overload flow [Pa]

$\Delta P_{\text {part }} \quad$ Inducer pressure head at part-load flow [Pa]

$\eta \quad$ Inducer efficiency

$\eta_{\text {opt }} \quad$ Inducer efficiency at optimal flow

$\eta_{\text {over }} \quad$ Inducer efficiency at overload flow

$\eta_{\text {part }} \quad$ Inducer efficiency at part-load flow

$\omega \quad$ Angular velocity $(2 \pi n / 60)[\mathrm{rad} / \mathrm{s}]$

$\mu \quad$ Dynamic viscosity [Pa.s]

$\rho \quad$ Fluid density, $\left[\mathrm{kg} / \mathrm{m}^{3}\right]$

$\sigma_{\mathrm{m}} \quad$ Mean solidity $\left(C_{\mathrm{m}} / s_{\mathrm{m}}\right)$

$\tau \quad$ Shaft toque [N m]

$\varphi \quad$ Sweep angle $\left[{ }^{\circ}\right]$

$\Upsilon \quad$ Specific delivery work $\left[\mathrm{m}^{2} / \mathrm{s}^{2}\right]$

\section{Abbreviations}

CFD Computational fluid dynamics

MRF Moving reference frame

NPSH Net positive suction head

NSGA Non-dominated sorting genetic algorithm

OPAL++ Optimization Algorithm Library++

RSM Reynolds stress model

SST Shear stress transport

\section{Introduction}

Centrifugal pumps are used in uncountable applications to transport single and two-phase flows. Be it for industrial or domestic use, its simple design, the broad range of flow rates and head, low maintenance requirement have made it an ideal machine for various applications. This includes petroleum industry (artificial lifting) (Caridad et al. 2008; Zhu et al. 2008; Monte Verde et al. 2017; Zhu et al. 2017), agriculture (irrigation) (Jiang et al. 2019), geothermal power stations (Amoresano et al. 2014), refrigeration (coolant pumping) (Si et al. 2018), nuclear power stations (emergency cooling systems) (Schäfer et al. 2017; Chan et al. 1999; Poullikkas 2003), chemical industries, medical treatment, paper industry, shipbuilding industry, food production, oil industry and waste-water treatments (Neumann et al. 2016; Cappellino et al. 1992).

Centrifugal pumps were initially designed for singlephase flows, providing excellent performance. However, it is a well-known fact that the pump performance and efficiency of a standard single-stage centrifugal pump decrease drastically under two-phase conditions as compared to single-phase, even at low gas volume fractions (Mansour et al. 2018a, b; Si et al. 2017). The reason is that the gas tends to accumulate within the impeller channels evolving radially as the gas volume fraction is increased. This makes the impeller unable to transfer kinetic energy to the two-phase mixture (Caridad et al. 2008; Jiang et al. 2019; Sato et al. 1996; Caridad and Kenyery 2004). For instance, at around 5\% gas content in volume, the gas pockets can be long enough to block the impeller channels, leading to a phenomenon known as "gas-locking" (Manzano 1980; Poullikkas 2003; Caridad et al. 2008; Campo and Chisely 2010; Mansour et al. 2018a, b). Furthermore, at around 7-10\% gas volume fractions, the impeller is completely blocked by gas pockets leading to "pump break-down", where the pump is unable to generate any head (Manzano 1980; Cappellino et al. 1992; Poullikkas 2003; Mansour et al. 2018a, b). Under certain gas flow conditions, there might be continuous formation and discharge of gas pockets within the impeller channels causing severe flow instabilities, vibrations, and unstable oscillations in pump performance parameters like flow rate, head, and efficiency. This phenomenon is known as pump "surging" (Tillack 1998; Sauer 2003; Gamboa and Prado 2011; Monte Verde et al. 2017; Zhu et al. 2017; Mansour et al. 2018a, b).

To improve the two-phase performance of centrifugal pumps, several studies confirmed that an increase in rotational speed raises the turbulence level, thereby creating a more homogeneous two-phase mixture. This improves the pump capability to handle higher gas volume fractions and delays the pump break-down (Schiavello 1986; Sauer 2003; Monte Verde et al. 2017; Mansour et al. 2018c; Kopparthy et al. 2020). Further studies found that a semi-open impeller provides a higher resistance to gas accumulation as compared to a closed impeller for a gas volume fraction in the range of $1-3 \%$. This is because, in semi-open impellers, the tip leakage flow (secondary flow) occurring within the impeller clearance gap helps to resist the gas agglomerations by improving two-phase mixing (Merry 1976; Cappellino et al. 1992; Furukawa et al. 1995; Mansour et al. 2018a; 
Hundshagen et al. 2019, 2021). Murakami and Minemura Murakami and Minemura (1974) showed that an impeller with 5 to 7 blades provides better performance for single and two-phase flow pumping compared to a 3-blade impeller. Sato et al. (1996) found that the impellers with a large blade outlet angle maintain the pump head when operating at higher gas volume fractions. Stel et al. (2019) used splitter-blade impeller to achieve higher mixing, reduce gas coalescence, and boost pump performance.

Multi-objective optimization has generally formed a strong foundation to tackle and optimize the design of various turbomachines (Sá et al. 2018, 2017; Song and Keane 2005; Nicholas et al. 2015; Rodrigues and Marta 2020; Chirkov et al. 2018). The design of pump impellers was also optimized in several studies using multi-objective optimization. For instance, Zhang et al. (2011) performed an optimization study of a helico-axial multiphase pump to improve efficiency and increase pressure rise. The optimized impeller showed an improvement in the pressure rise and the efficiency by $10 \%$ and $3 \%$, respectively, compared to the original impeller. Safikhani et al. (2011) focused on two conflicting objectives, i.e., increasing the efficiency and simultaneously decreasing the net positive suction head required $\left(\mathrm{NPSH}_{\mathrm{r}}\right)$ of the pump. In their study, they found optimal combinations of the impeller angles for these objectives. Huang et al. (2015) performed a Pareto-based optimization with 10 design variables to control the blade loading with the aim of improving the hydraulic efficiency together with the impeller head of a mixed-flow pump. Their optimized impellers showed improved performance with a high-efficiency operating range. Nariman-Zadeh et al. (2007) performed an optimization study considering the flow rate and the impeller radius as input parameters, obtaining efficient designs that show an increase in head and efficiency along with a decrease in input power. Han et al. (2020) performed a centrifugal pump impeller and volute shape optimization via genetic algorithms and back propagation neural network. Impeller head and efficiency could be increased by $7.69 \%$ and $4.74 \%$ while the power consumption decreased by $2.56 \%$ post-optimization. Another interesting study focused on improving the performance and reducing the energy consumption of a centrifugal water pump to carry out slurry flows (Derakhshan and Bashiri 2018). The maximum improvement in the efficiency was found around $4 \%$ by increasing $9.9 \%$ of the head. According to these studies, it can be concluded that optimizing the impeller geometrical parameters and the flow conditions have been already often considered in the literature, achieving performance improvements.

Additional studies have shown that inducers, simple axialflow impellers generally employed upstream of the main centrifugal pump, improve pump cavitation performance by reducing the net positive suction head required. This improvement is due to the increased pressure of the flow transmitted toward the pump impeller (Sulzer 2013; Gülich 2008; Hong et al. 2006; d'Agostino et al. 2017; CamposAmezcua et al. 2013; Pouffary et al. 2008; Bakir et al. 2004; Guo et al. 2015; Lundgreen et al. 2019; Song et al. 2016; Oshima 1967; Choi et al. 2007). Additionally, several studies investigated the impact of various geometrical parameters on cavitation behavior and performance. For instance, Fu et al. (2017), Kim et al. (2017) and Ji et al. (2020) showed that an increase in the tip clearance can generally lead to larger hydraulic losses at low-flow rates. El Samanody et al. (2014) investigated inducers with helical or axial blades by changing several parameters, i.e., pitch, number of blades, number of turns, inlet and outlet blade angles, shaft length, and shaft diameter. It was shown that a two-turn helical-blade inducer with a $17^{\circ}$ helical angle resulted in the best performance at high rotational speeds, while the performance of an axial blade inducer with three or five blades is better at low rotational speeds. Guo et al. (2016) showed that the cavitation performance of a pump with a 3-bladed inducer is better than those with 2- and 4-bladed inducers. Apart from improving the cavitation performance of a pump, inducers have also been known to improve the two-phase handling capability of centrifugal pumps by providing a more homogeneous mixture at the pump inlet (Cappellino et al. 1992; Mansour et al. 2018b; Thum 2007; Mansour et al. 2019, 2020a; Parikh et al. 2020; Mansour et al. 2020b).

In our previous studies on single-phase and two-phase flows involving inducers (Mansour et al. 2020a, 2019), the impact of the numerical settings and various turbulence models have been studied, with the objective of an accurate description of all flow properties around the inducer. It was eventually found out that the Reynolds stress model (RSM) was able to accurately reproduce all flow features, providing excellent agreement with experimental data. As a faster alternative, the $k-\omega$ shear stress transport (SST) model was also able to deliver a fair agreement with all measurements. In Mansour et al. (2019), the primary goal was to understand the reason for the dissimilar effects of the inducer on two-phase pumping performance at part-load and overload conditions, with a significantly higher influence at partload (Mansour et al. 2018b, 2019; Parikh et al. 2020). This numerical study revealed the formation of strong, axially propagating vortices starting from the blade leading edge and extending all along the inducer length, increasingly so with increasing flow rates. This explained the negative performance at overload conditions. In Mansour et al. (2020a), the investigations were extended to two-phase conditions. The numerical results showed that a much higher two-phase mixing can be achieved at part-load because of the long residence time, typically available for low-flow rates. This explains why this inducer can only noticeably improve twophase pumping performance at part-load. Nevertheless, it 
must be kept in mind that both studies (Mansour et al. 2019, 2020a) considered only a single inducer design, with a pitch of $P=0.251 \mathrm{~m}$ and a number of blades of $N=3$.

Shojaeefard et al. (2019) took a more systematic approach to obtain an optimized inducer design by using a multiobjective optimization technique (NSGA-II, Non-dominated Sorting Genetic Algorithm-II). Several inducer design parameters like the inlet and outlet tip blade angle and the ratio of outlet hub radius to the inlet hub radius were varied to optimize the inducer performance. A noticeable improvement of $14.3 \%, 0.3 \%$ and $30.2 \%$ was obtained for inducer head coefficient, hydraulic efficiency, and net positive suction head, respectively. Additionally, the neural network was used to model the objective functions with respect to the input parameters. This study particularly shows that certain optimum design parameters can only be obtained using a multi-objective optimization approach. Although this study has explored and optimized some of the inducer geometrical parameters, the effect of a wide number of other geometrical parameters on the inducer performance like the number of blades and pitch among others remains unexplored in the uncharted territory.

In a first effort to understand the influence of inducer's geometry on single and two-phase flows, our recent study (Mansour et al. 2020b) involved three different inducer pitches $(P=0.151 \mathrm{~m}, 0.251 \mathrm{~m}$, and $0.351 \mathrm{~m})$ and three different blade numbers ( $N=2,3$ and 4), resulting in 9 different configurations. This study showed a significant impact of both geometrical parameters on the inducer performance, the flow vortices, and the two-phase mixing behavior. The results revealed that under single-phase conditions, inducers with a low solidity, i.e., a high pitch and a low number of blades, provide better performance at overload conditions since they only lead to weak vortical structures. However, an inducer with a high solidity should be preferred at partload conditions. At the same time, a higher number of blades resulted in a slightly decreased inducer peak efficiency and reduced the effective flow range. Concerning two-phase flows, an inducer with a higher pitch is able to more efficiently churn the two phases, providing better mixing. A higher number of blades resulted in a slight improvement in two-phase mixing at high flow conditions. Accordingly, inducers with a higher pitch and a low to moderate number of blades were recommended to ensure high single-phase as well as two-phase performances with effective mixing, and a wide range of effective operation.

From all previous studies, it can be concluded that the influence of the geometrical parameters of the inducer is very large - and quite complex, with many cross-dependencies and contradictory statements regarding overall performance. The observations differ for single-phase and two-phase, for part-load and overload, regarding peak efficiency or usable flow range. Additionally, most of the previous optimization studies considered only isolated geometrical parameters, a constant flow rate, a single objective function representing the performance, or combinations of those limitations. This explains the need for a far more systematic study, considering simultaneously:

- All relevant geometrical parameters describing inducer design;

- Different flow rates covering part-load, optimal, and overload conditions;

- All important properties quantifying "performance" for the considered process.

For this reason, the present study involves three different, normalized flow rates, i.e., $Q / Q_{\mathrm{opt}}=0.8,1.0$, and 1.5 , where $Q_{\mathrm{opt}}$ is the optimal (design) flow rate of the pump. The objective of the present optimization is to maximize simultaneously the efficiency as well as the pressure head curves obtained with the corresponding inducer; this is the performance indicator. Finally, a total of 8 geometrical parameters have been varied, including axial blade length $(L)$, blade sweep angle $(\phi)$, blade pitch $(P)$, hub taper angle $(\beta)$, tip clearance gap $(C)$, blade thickness at the hub $\left(T_{\mathrm{h}}\right)$, blade thickness at the tip $\left(T_{\mathrm{t}}\right)$, number of blades $(N)$, as illustrated later in Fig. 4. In this way, optimal inducer configurations for a wide range of flow rates can be obtained. Due to the complexity of the resulting optimization problem, the present study considers only single-phase flows (liquid water). Extending toward two-phase flows will be the subject of the next investigation.

The optimization process was done by employing a fully automatized in-house optimization library (the Optimization Algorithm Library++, written shortly OPAL++, Daróczy et al. (2014)) to control the CFD simulations. An efficient multi-objective global optimization method [NSGA-II, Nondominated Sorting Genetic Algorithm-II, Deb et al. (2002)] was applied. A total of 37 generations involving 5178 different CFD simulations were performed during the optimization process. In this manner, a Pareto front containing 5 optimal inducer configurations was obtained for the objective functions. The influence of each design parameter on the objective functions is discussed in the upcoming sections. In particular, it is found that a high hub taper angle with 3 blades should be used to maximize inducer performance, while blade length, blade sweep angle, tip clearance gap, and blade thickness should be kept low.

\section{Methodology}

\subsection{Numerical modeling}

The industrial CFD code Siemens STAR-CCM+ (2018) was used to perform all CFD simulations. It solves the 
continuity and momentum equations as given by Eqs. (1) and (2), respectively, based on the finite-volume method.

$\frac{\partial \rho}{\partial t}+\nabla \cdot(\rho \mathbf{V})=0$

$\rho \frac{D \mathbf{V}}{D t}=\rho \mathbf{g}-\boldsymbol{\nabla} \cdot p+\mu \nabla^{2} \cdot \mathbf{V}$

where $\rho$ is the fluid density, $\mathbf{V}$ is the fluid velocity vector, $\mathbf{g}$ is the gravitational acceleration, $p$ is the pressure, and $\mu$ is the fluid viscosity. Further details about the governing equations can be found in STAR-CCM+ (2018). It was already demonstrated in a previous study (Mansour et al. 2019) that the numerical results obtained with either (1) an unsteady solver with the moving mesh approach or (2) a steady-state solver with the Moving Reference Frame (MRF) are very comparable for the present configuration; however, the latter approach is much faster in terms of computing time, and must therefore be preferred for an optimization. Thus, the steady-state solver with MRF was used in the present analysis to model the rotation of the inducer; MRF is also known as the "frozen-rotor approach", where a constant grid flux corresponding to the Coriolis force is introduced in the source term of the conservation equations, mimicking the real movement of the rotor. These modified conservation equations are solved within a rotating region where the inducer is placed, representing the inducer rotation. To enable comparisons with previous studies (Mansour et al. 2019, 2020a; Parikh et al. 2020; Mansour et al. 2020b), a constant rotation rate of $650 \mathrm{rpm}$ was again used in the present analysis. Furthermore, a mass flow inlet boundary condition was given on the domain inlet, corresponding to the desired load, i.e., part-load, optimal, or overload conditions. Though a constant rotational speed was kept, the results of this optimization should also be valid at other rotational speeds, since the study was done based on a non-dimensional analysis for single-phase conditions; the performance curves of pumps (and inducers) at different rotational speeds become identical when plotted on non-dimensional (normalized) scales for single-phase flows, based on the pump affinity laws. However, this is not always true for two-phase flows, since the effect of the gas on the pump performance at a specific gas volume fraction changes also with the rotational speed.

Our previous study (Mansour et al. 2019) showed that turbulence can be accurately modeled using either the Reynolds Stress Model (RSM) with second-order quadratic pressure strain or the $k-\omega$ shear stress transport (SST) model. The model formulation of the Reynolds Stress Model (RSM) can be found in Sarkar and Balakrishnan (1990) and Speziale et al. (1991), while the details of the $k-\omega$ shear stress transport (SST) model are available in Menter (1994). A comparison showing the normalized pressure head obtained by the RSM model, the $k-\omega$ model, and the corresponding experimental data for the original, standard inducer is shown in Fig. 1. Both turbulence models show very good agreement with the experimental data along the whole flow range. Unlike the $k-\omega$ turbulence model, which is a simple two-equation model, the RSM model solves six different equations to model turbulence, making it computationally very expensive. As a consequence, the $k-\omega$ (SST) model was preferred for the present optimization study to ensure acceptable computational efforts. Water was modeled as an incompressible fluid with a density of $\rho=998.2 \mathrm{~kg} / \mathrm{m}^{3}$ and dynamic viscosity of $\mu=1.00310^{-3} \mathrm{~Pa} \cdot \mathrm{s}$. To ensure good computational accuracy, a second-order upwind discretization scheme was applied for computing the convection flux on cell faces. The numerical simulations were stopped when the residuals reached $10^{-4}$ (absolute), or a drop of three orders of magnitude compared to the initial values (relative); a few a posteriori checks have confirmed that this is sufficient to ensure convergence. Additional details concerning the numerical settings have been kept identical to similar studies (Mansour et al. 2019, 2020a).

\subsection{Simulation domain and meshing}

Figure 2 shows the numerical simulation domain used for the present study. The inducer geometry shown in Fig. 2 corresponds to the original prototype inducer used experimentally and numerically in several of our previous publications (Mansour et al. 2018b, 2020a; Parikh et al. 2020; Mansour et al. 2019, 2020b). All the dimensions are given as a function of the suction pipe diameter $(d)$, which is kept constant for all simulations. The corresponding geometrical specifications of the original inducer are listed in Table 1. As seen, the domain is divided into two equal parts, i.e., the stationary domain and the rotating domain surrounding the inducer. The two domains are connected via an in-place

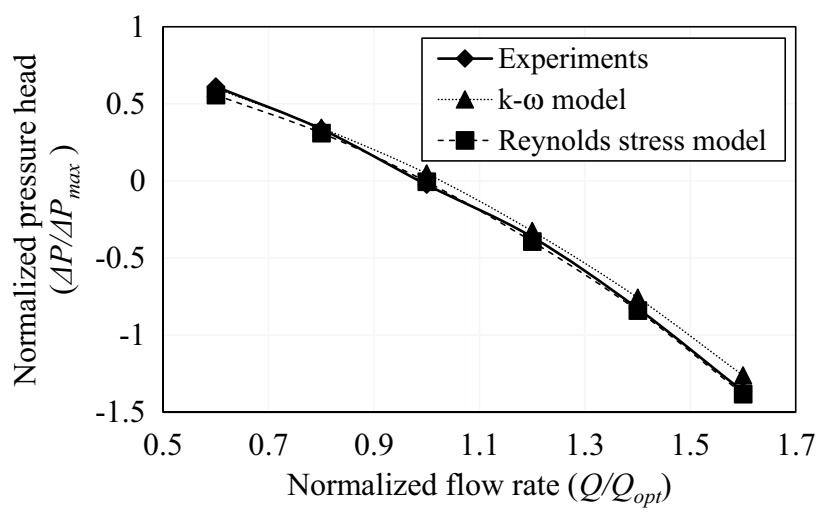

Fig. 1 Comparison between the normalized pressure head obtained by RSM model, $\mathrm{k}-\omega$ model, and the experiments 


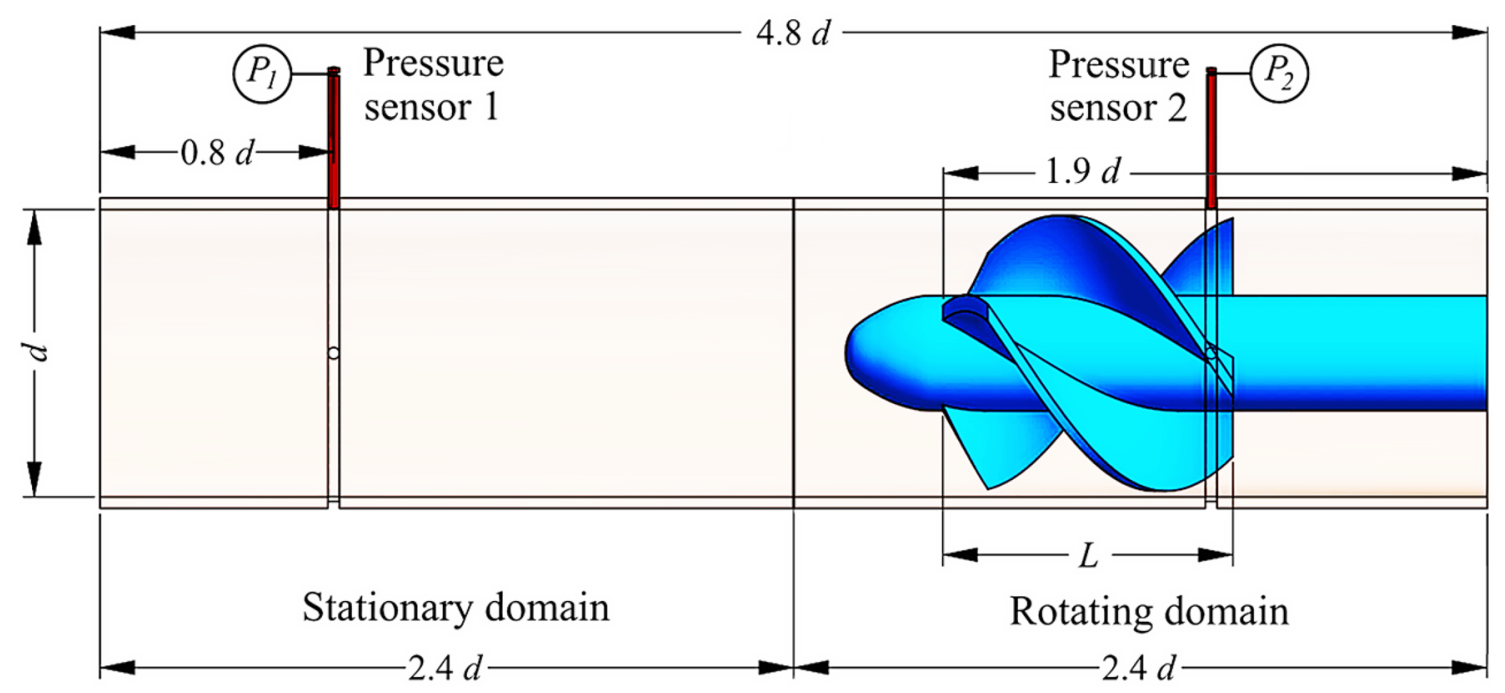

Fig. 2 Details of the simulation domain with the original, standard inducer

interface to transfer the numerical information. A no-slip adiabatic boundary condition was applied along all walls. Additionally, the walls surrounding the rotating domain were modeled as stationary walls by keeping the tangential velocity zero. A uniform velocity profile was applied at the inlet section of the simulation domain, while a constant-pressure boundary condition was always used at the outlet surface. Two pressure sensors $P_{1}$ and $P_{2}$ are placed within the simulation, upstream and downstream of the inducer, corresponding to the real locations in the experiments used for head measurements. These CFD "pressure sensors" measure the circumferential arithmetic average pressure, similar to the experiments (several holes are drilled along the circumference of the tube and connected together). Note that pressure sensor 2 is installed directly at the end of the inducer blades because it was not possible to move it further downstream in the experiments since the impeller is installed directly behind the inducer (Mansour et al. 2019, 2020a) (different from Fig. 2, where no centrifugal pump is involved). In all CFD simulations, this numerical sensor was always placed at the end of the blades for each inducer configuration (similar to the experiments) by shifting according to the blade length $L$.

Figure 3 shows a sample view of the numerical grid used in the present study generated using the automatic polyhedral meshing tool available in the CFD code Siemens STAR-CCM+. Grid type and necessary resolution have been extensively tested and validated in previous similar studies (Mansour et al. 2019, 2020a). To ensure accurate boundary layer modeling, 8 prism layers were always used near all walls. The thickness of the first layer was kept sufficiently small to ensure an average non-dimensional wall distance $\left(y^{+}\right)$less than 1 . Additionally, sufficient refinement was ensured within the rotating domain around the inducer body to accurately resolve all flow features within the zone of interest (Mansour et al. 2019, 2020a). The mesh shown in Fig. 3 contains approximately 3.25 million polyhedral elements and has an average $y^{+}$value of 0.3 . The same mesh settings were used to maintain similar resolution in space and $y^{+}$values for all inducer configurations considered during optimization. Additional details regarding mesh quality can be found in Mansour et al. $(2019,2020 \mathrm{a})$.
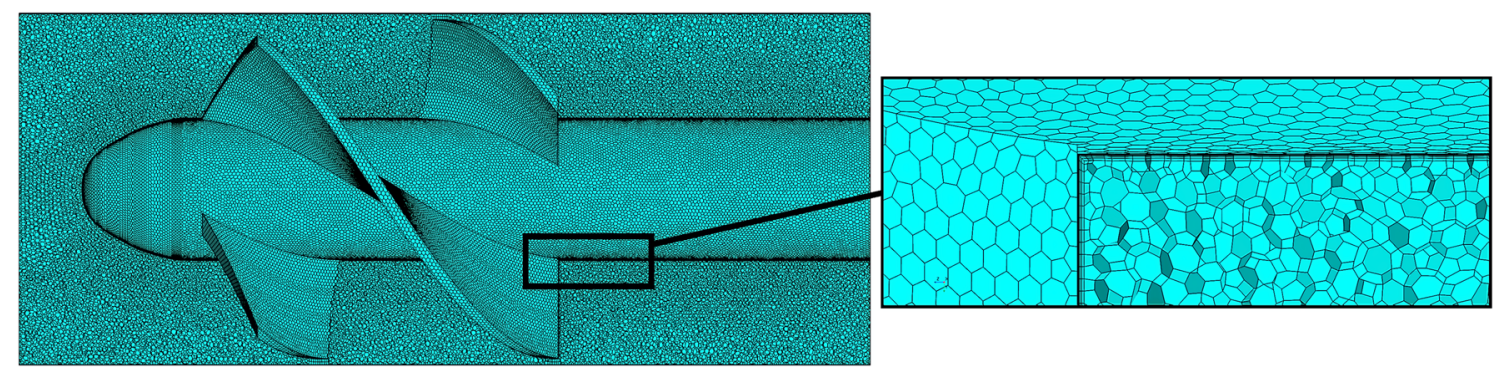

Fig. 3 A sample view of the employed mesh (approximately involving 3.25 million cells) for the original, standard inducer 


\subsection{Design parameters}

As mentioned in the introduction, a total of 8 important geometrical parameters have been considered, which are the axial blade length $(L)$, the blade sweep angle $(\varphi)$, the blade (helical) pitch $(P)$, the hub taper angle $(\beta)$, the tip clearance gap $(C)$, the blade thickness at the hub $\left(T_{\mathrm{h}}\right)$, the blade thickness at the tip $\left(T_{\mathrm{t}}\right)$, and the number of blades $(N)$. Figure 4 shows all the considered geometrical parameters of a representative inducer. Note that the axial blade pitch $(P)$ can be defined as the axial distance of one complete helical turn of the blade. The distance $P / N$ can be represented in the warped view of the inducer as shown in Fig. $4 \mathrm{~b}$ where each parallel pair of oblique lines represent a blade of the inducer with a specific thickness as shown.

The upper and lower limits for each design parameter have been carefully selected based on previous studies (Mansour et al. 2020b; Gülich 2008; Fu et al. 2017; Kim et al. 2017; El Samanody et al. 2014; Guo et al. 2016). Corresponding values are listed in Table 1, after normalization using the pipe diameter $(d)$ whenever possible.
According to those ranges, a wide diversity of inducer configurations will be generated, avoiding invalid designs.

Additionally, the resulting ranges for some derived, significant geometrical parameters of the inducer have been calculated, normalized, and included as well in Table 1, in particular, the mean hub diameter $\left(D_{\mathrm{h}}\right)$, the blade tip diameter $\left(D_{\mathrm{t}}\right)$, the mean chord length $\left(C_{\mathrm{m}}\right)$, the blade helix angle $(\theta)$, and the mean solidity of the inducer $\left(\sigma_{\mathrm{m}}\right)$. These parameters will be used in the analysis of the results to provide a better understanding of the optimal configurations. Figure 4 illustrates also $D_{\mathrm{h}}, D_{\mathrm{t}}$, and $C_{\mathrm{m}}$. Note that the mean hub diameter $\left(D_{\mathrm{h}}\right)$ is calculated at the mid-axial length $(0.5 L)$ of the inducer. The mean inducer solidity can be defined by Equation (3), where $C_{\mathrm{m}}$ is the mean chord length of the blades and $s_{\mathrm{m}}$ is the mean azimuthal blade spacing as given by Eqs. 4 and 5, respectively. As illustrated in Fig. 4b, these parameters are defined based on the mean blade diameter $D_{\mathrm{m}}$, which can be determined by Eq. (6), where $D_{\mathrm{h}}$ and $D_{\mathrm{t}}$ can be calculated from Eqs. 7 and 8 , respectively. Finally, the blade helix angle $\theta$, shown in Fig. 4b, can defined by Eq. 9 .

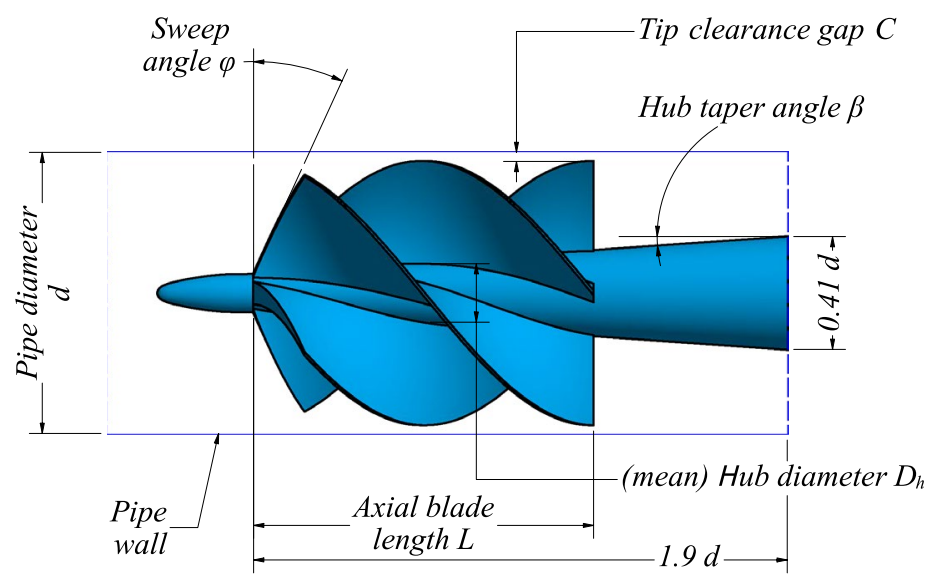

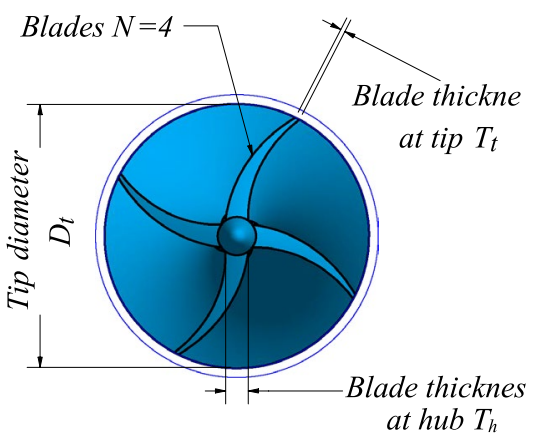

Front view

(a) Geometrical design parameters of a representative inducer geometry.

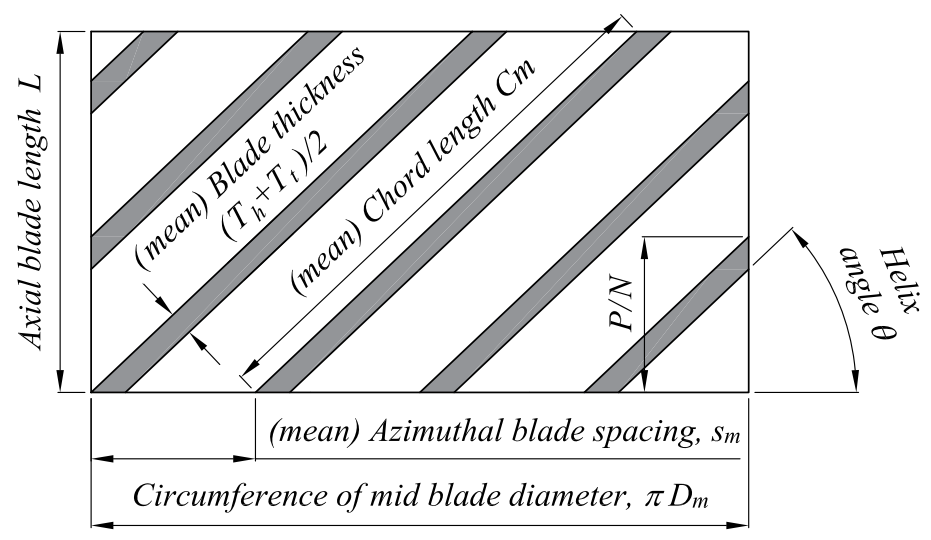

(b) Warped view of a representative inducer geometry.

Fig. 4 Illustration of the input design parameters of the optimization process 
Table 1 Ranges of design parameters for the optimization

\begin{tabular}{llllll}
\hline$\#$ & Design parameters & & Lower limit & Original inducer & Upper limit \\
\hline 1 & Normalized axial blade length & $L / d$ & 0.8 & 1.0 & 1.41 \\
2 & Blade sweep angle & $\varphi\left[^{\circ}\right]$ & 0 & 29.4 & 60 \\
3 & Normalized blade pitch & $P / d$ & 2.0 & 2.0 & 8.1 \\
4 & Hub taper angle & $\beta\left[^{\circ}\right]$ & 0 & 0 & 5.5 \\
5 & Normalized tip clearance gap & $C / d$ & 0.008 & 0.02 & 0.081 \\
6 & Normalized blade thickness at hub & $T_{\mathrm{h}} / d$ & 0.016 & 0.0622 & 0.0726 \\
7 & Normalized blade thickness at tip & $T_{\mathrm{t}} / d$ & 0.016 & 3 & 0.081 \\
8 & Number of blades & $N$ & 1 & Original inducer (Mansour et al. & Upper limit \\
\hline$\#$ & Derived parameters & & $2019,2020 \mathrm{a})$ & \\
\hline 1 & & & Lower limit & 0.402 & 0.96 \\
2 & Normalized mean hub diameter & $D_{\mathrm{h}} / d$ & 0.115 & 0.402 \\
3 & Normalized tip diameter & $D_{\mathrm{t}} / d$ & 0.84 & 0.984 \\
4 & Normalized mean chord length & $C_{\mathrm{m}} / d$ & 0.83 & 4.466 & 7.7 \\
5 & Blade helix angle & $\theta\left[^{\circ}\right]$ & 43 & 2.056 & 3.46 \\
\hline
\end{tabular}

$\sigma_{\mathrm{m}}=\frac{C_{\mathrm{m}}}{s_{\mathrm{m}}}$

$C_{\mathrm{m}}=L \sqrt{1+\left(\frac{\pi \cdot D_{\mathrm{m}}}{P}\right)^{2}}$

$s_{\mathrm{m}}=\frac{\pi \cdot D_{\mathrm{m}}}{N}$

$D_{\mathrm{m}}=\frac{D_{\mathrm{h}}+D_{\mathrm{t}}}{2}$

$D_{\mathrm{h}}=0.41 d+(L-3.8 d) \tan \beta$

$D_{\mathrm{t}}=d-2 C$

$\theta=\tan ^{-1}\left(\frac{P}{\pi \cdot D_{\mathrm{m}}}\right)$

Figure 5 presents sample inducers corresponding to the lower (left) and upper (right) limits of each design parameter, taking the original prototype inducer, used in previous studies (Mansour et al. 2019, 2020a), as the base geometry. As a complement, Fig. 6 depicts arbitrarily selected inducer geometries demonstrating the diversity of the designs considered during the optimization study.

\subsection{Optimization objectives}

The objective of the present study is to optimize the inducer geometry by maximizing simultaneously the area under the pressure head and efficiency curves, and this for three different flow rates. Accordingly, for each single inducer configuration, simulations at $Q / Q_{\mathrm{opt}}=0.8$ (part-load), $Q / Q_{\mathrm{opt}}=1.0$ (nominal), and $Q / Q_{\text {opt }}=1.5$ (overload) have been carried out, where $Q_{\text {opt }}$ is the optimal flow rate of the pump (Mansour et al. 2018b). In this way, optimal inducer configurations for a wide flow range can be obtained. The inducer efficiency is defined by Eq. (10) which represents the ratio of the output fluid power from the inducer $\left(P_{\mathrm{f}}\right)$ to the shaft power $\left(P_{s h}\right)$. Here $\dot{m}$ is the mass flow rate, $\Upsilon$ is the specific work of the inducer, $\tau$ is the shaft torque and $\omega$ is the angular speed.

$\eta=\frac{P_{\mathrm{f}}}{P_{s h}}=\frac{\dot{m} \cdot \Upsilon}{\tau \cdot \omega}$

The torque $(\tau)$ is calculated in the simulations by Eq. (11), where $r$ represents the position of face $f$ relative to the axis $a$ about which the torque is calculated, $F_{\mathrm{P}}$ is the pressure force, and $F_{\mathrm{S}}$ is the shear force. The specific delivery work $(\Upsilon)$ is calculated by Eq. (12). Here, $V_{1}$ is the fluid velocity at pressure sensor $1, V_{2}$ is fluid velocity at pressure sensor $2, g$ is the gravitational acceleration, $z_{1}$ is the elevation at pressure sensor 1 , and $z_{2}$ is the elevation at pressure sensor 2. The complete derivation of Eq. (12) can be found 


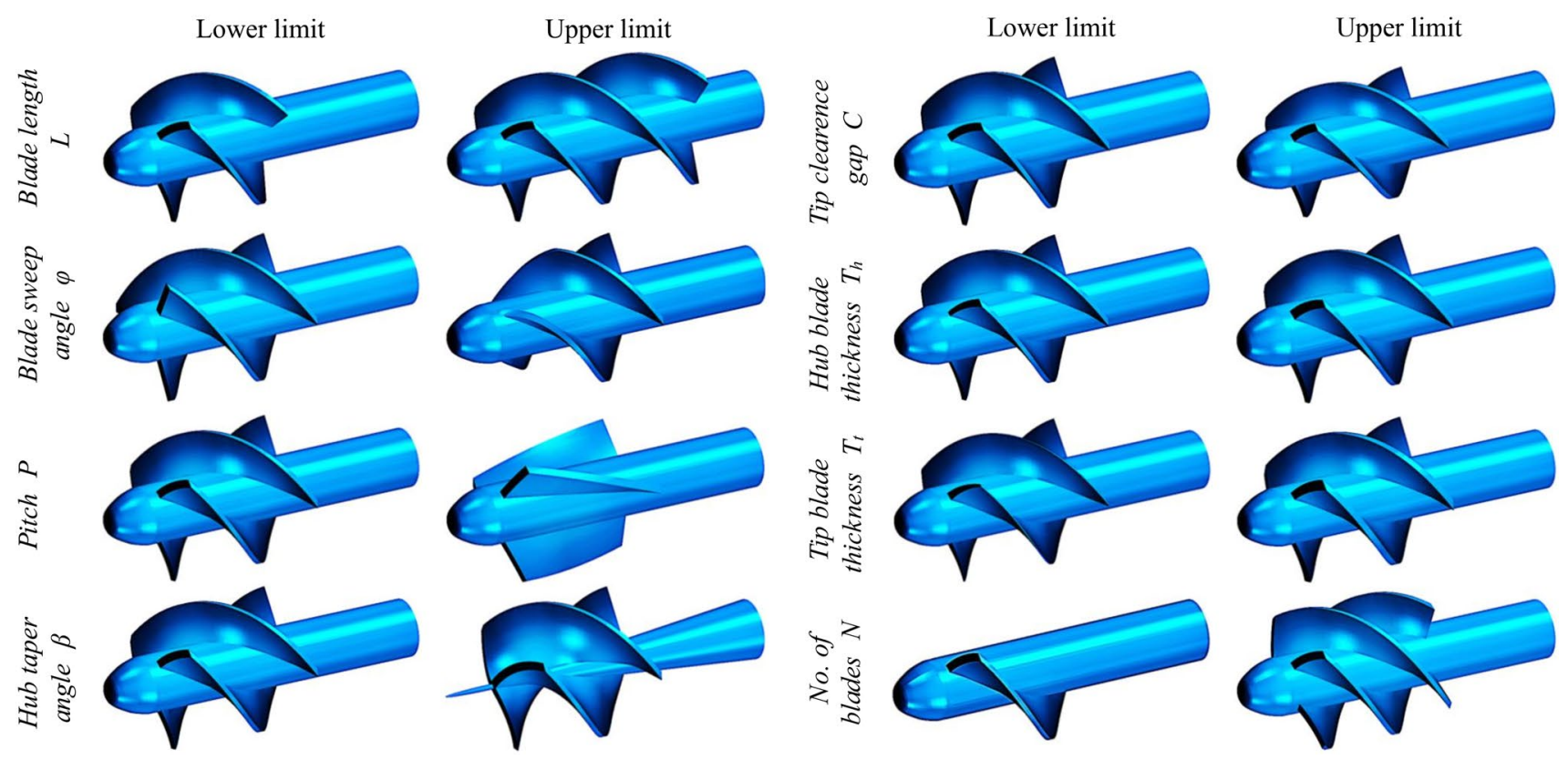

Fig. 5 Visualization of the inducer geometry corresponding to the upper and lower limits of all design parameters considered in the optimization study
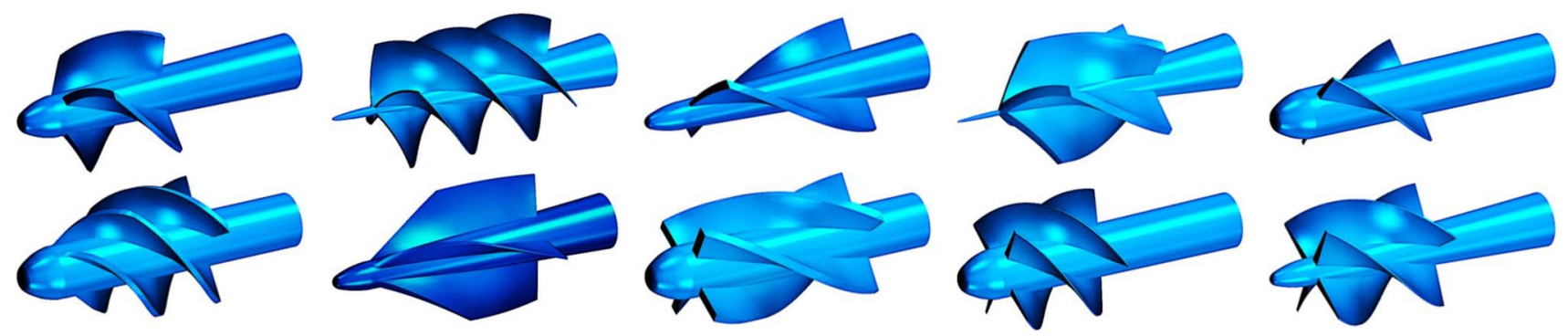

Fig. 6 Arbitrarily-chosen inducer geometries illustrating the diversity of all designs involved in the optimization study

in Mansour et al. (2018a). The fluid velocities $\left(V_{1}\right.$ and $\left.V_{2}\right)$ are calculated based on the continuity equation as given by Eqs. (13) and (14), dividing the fluid flow rate $(Q)$ by the pipe cross-sectional area at pressure sensor $1\left(A_{1}\right)$ and pressure sensor $2\left(A_{2}\right)$, respectively.

$$
\begin{aligned}
\tau= & \sum_{f}\left[r \times\left(F_{\mathrm{P}}+F_{\mathrm{S}}\right)\right] \cdot a \\
\Upsilon= & \frac{\left(P_{2}-P_{1}\right)}{\rho}+\frac{1}{2}\left(V_{2}^{2}-V_{1}^{2}\right) \\
& +g\left(z_{2}-z_{1}\right) \\
V_{1}= & \frac{Q}{A_{1}}
\end{aligned}
$$

$V_{2}=\frac{Q}{A_{2}}$

Figures $7 \mathrm{a}$ and $\mathrm{b}$ illustrate the calculations of the area under the pressure head $\left(A_{\Delta P}\right)$ and the efficiency $\left(A_{\eta}\right)$ for the considered flow rates. As shown, the output values of the three different simulations are used to calculate the (approximated) area under each curve, which are given using the trapezoidal rule by Eqs. (15) and (16), respectively. Here $\Delta P_{\text {part }}$ is the pressure head at part-load flow $\left(Q / Q_{\mathrm{opt}}=0.8\right), \eta_{\mathrm{part}}$ is the efficiency at part-load flow $\left(Q / Q_{\text {opt }}=0.8\right), \Delta P_{\text {opt }}$ is the pressure head at optimal (or nominal) flow $\left(Q / Q_{\mathrm{opt}}=1.0\right), \eta_{\mathrm{opt}}$ is the efficiency at optimal flow $\left(Q / Q_{\text {opt }}=1.0\right), \Delta P_{\text {over }}$ is the pressure head at overload flow $\left(Q / Q_{\text {opt }}=1.5\right)$, and $\eta_{\text {over }}$ is the efficiency at overload flow $\left(Q / Q_{\mathrm{opt}}=1.5\right)$. Note that the selected part-load flow is not deep part-load, since it has been shown that the inducer geometry does not impact much performance at very low-flow rates (Mansour et al. 2020b). 
Fig. 7 Objective functions $\left(A_{\Delta P}\right.$ and $A_{\eta}$ ) of the optimization process

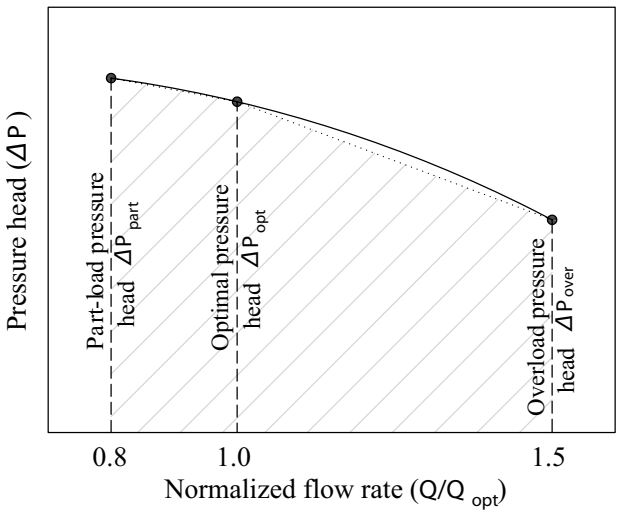

(a) Objective function 1; area under pressure head curve $\left(A_{\Delta P}\right)$, approximated by trapezoidal rule (hatched area).

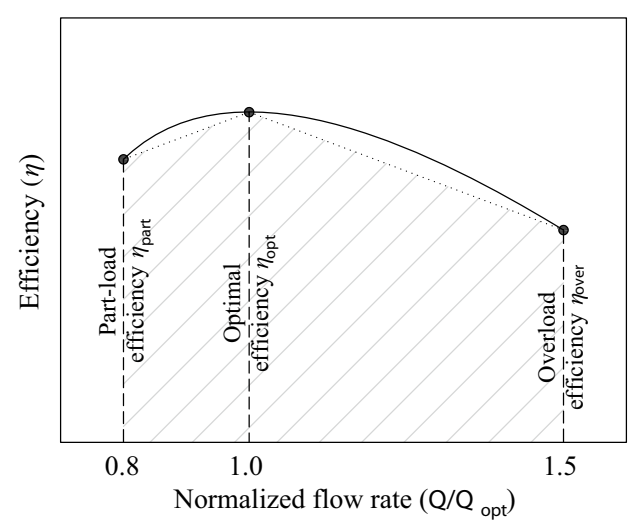

(b) Objective function 2; area under efficiency curve $\left(A_{\eta}\right)$, approximated by trapezoidal rule (hatched area).
$A_{\Delta P}=\left(0.1 \Delta P_{\text {part }}+0.35 \Delta P_{\text {opt }}+0.25 \Delta P_{\text {over }}\right)$

$A_{\eta}=\left(0.1 \eta_{\text {part }}+0.35 \eta_{\text {opt }}+0.25 \eta_{\text {over }}\right)$

\subsection{Statement of the optimization problem}

The present optimization problem can be stated as follows:

Find $X=\left\{\begin{array}{c}L / d \\ \varphi \\ P / d \\ \beta \\ C / d \\ T_{\mathrm{h}} / d \\ T_{\mathrm{t}} / d \\ N\end{array}\right\}$ which maximizes $f_{1}(X)=A_{\Delta P}$ and $f_{2}(X)=A_{\eta}$

where $X$ is an 8-dimensional design vector, i.e., containing 8 design variables, and $f_{1}(X)=A_{\Delta P}$ and $f_{2}(X)=A_{\eta}$ are the objective functions to be maximized.

\subsection{Multi-objective optimization}

The main task of the optimization is in the present case to maximize simultaneously the objective functions $A_{\Delta P}$ and $A_{\eta}$ by adapting appropriately the input design parameters within specified ranges. In the present optimization process, the two objective functions are considered to be equally important, i.e., no weighting functions were applied. A Pareto-based optimal approach was used together with the Non-dominated Sorting Genetic Algorithm (NSGA-II) (Deb et al. 2002) to optimize the inducer configuration. The Pareto-optimality approach tries to find the best set of solutions considering at the same time all objective functions, which is usually called the Pareto-optimal set. A solution is considered Pareto-optimal if it is not dominated by any other solution. This means that a Pareto-optimal solution cannot be improved further in any objective without simultaneously worsening at least one other objective function. The line connecting all optimal solutions is called the Pareto front.

The process starts with an initial guess (combinations of the design parameters), which is called the first generation. As a function of their respective objective functions, these designs are then used as a basis for cross-over and mutation, generating new individuals (i.e., new combinations of design parameters) for the next generation (Thévenin and Janiga 2008). This process continues until the obtained Pareto-optimal set cannot be noticeably improved any more. A fully automatized in-house optimization code (the Optimization Algorithm Library++, written shortly as OPAL++) was used to automatically change the design parameters and control the numerical simulations. This code has been continuously developed in our research group for the past 20 years. The current code is described in detail in Daróczy et al. (2014). Many successful design optimization studies have been carried out using OPAL++, for instance, Mansour et al. (2020c), Daróczy et al. (2014, 2016, 2018) and Kerikous and Thévenin (2019)), to cite a few.

Figure 8 illustrates the automatic optimization loop that has been used in the study. Firstly, OPAL++ generates an initial guess for the design variables, which is then written in a Java script containing all other settings to automatize the CFD simulation, done by Star-CCM+. Using the macro file, the simulations are run on a High-Performance Cluster (HPC). The Java script commands the CFD solver to create the simulation files, create a new inducer geometry based on the values written by OPAL ++ , generate a mesh, perform CFD simulations for the three different flow conditions, and perform post-processing by computing the objective values. The two objective functions $\left(A_{\Delta P}\right.$ and $\left.A_{\eta}\right)$ are calculated and exported in a separate output file. OPAL++ then reads the results and analyzes the data to generate the 


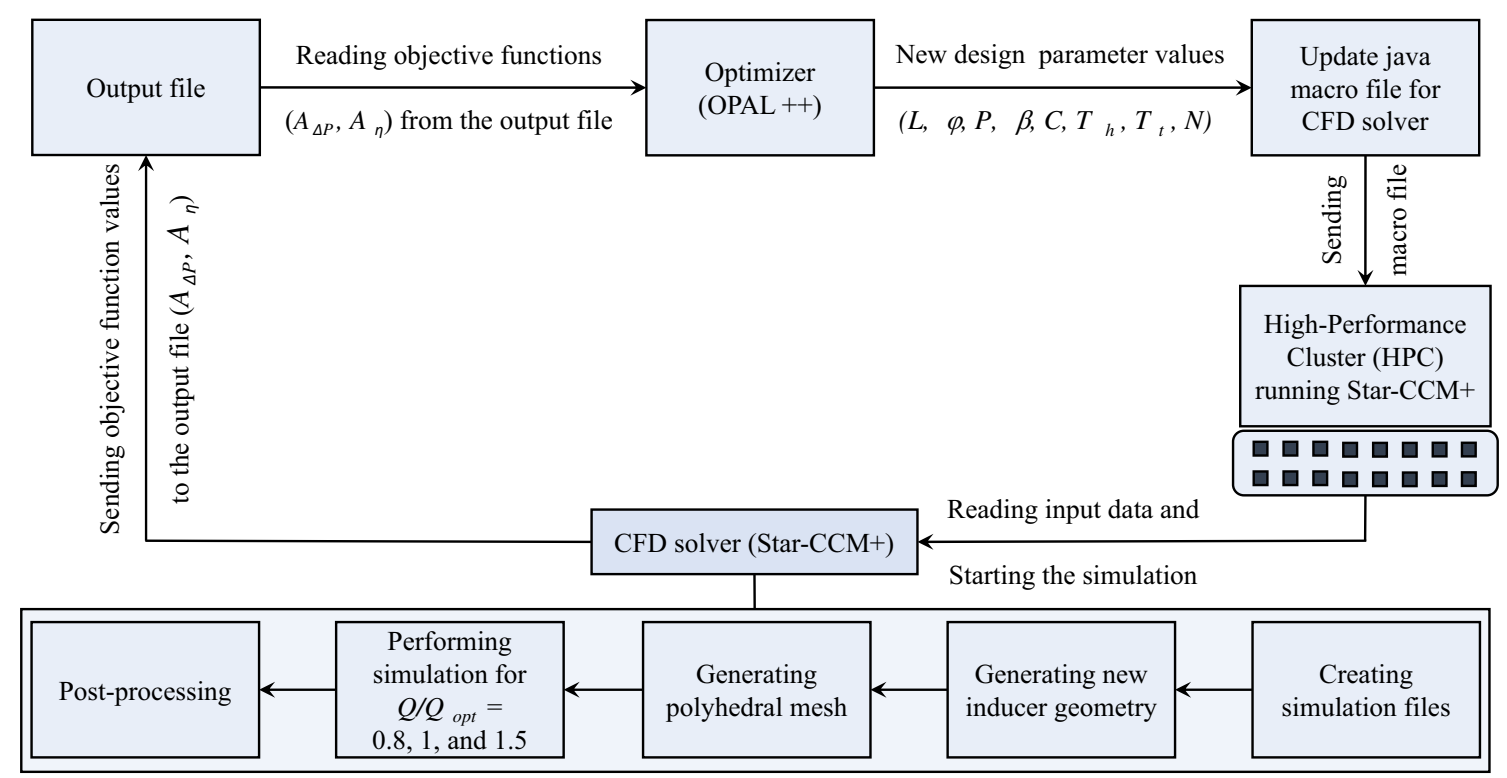

Fig. 8 Illustration of the automatic optimization loop

next generation of design variables thanks to cross-over and mutation. Concerning the first generation, a Sobol-based pseudo-random generator was used to initialize the first population with $N_{i}=48$ individuals, which is kept constant for the entire optimization process (dominated individuals with low objective functions being eliminated). The mutation probability and the cross-over probability were set to $1 / n_{\mathrm{d}}$ and 0.8 , respectively, following recommendations from (Deb et al. 2002), where $n_{\mathrm{d}}$ represents the number of design parameters. The computation time of a single generation (i.e., 48 CFD simulations) is about 12 hours wall-clock-time using 20 parallel nodes with 16 cores each. At the end of the optimization process, a total of 37 generations covering 5178 successful CFD simulations (including 1726 individuals with 3 simulations per individual) have been carried out in the present analysis.

\section{Results and discussion}

\subsection{General analysis}

The overall features of all configurations are first examined by using parallel coordinates. Such plots are commonly used to inspect high-dimensional problems using the simplified form of a 2D plot (Inselberg 2009; Edsall 2003; Kipouros et al. 2013). Figure 9 presents the data of all computed inducers in the form of such parallel coordinates, where the vertical lines represent separate scales for each quantity. The plot shows the eight design variables ( $\left.L / d, \varphi, P / d, \beta, C / d, T_{\mathrm{h}} / d, T_{\mathrm{t}} / d, N\right)$ on the left, then the five derived variables $\left(D_{\mathrm{h}} / d, D_{\mathrm{t}} / d, C_{\mathrm{m}} / d, \theta, \sigma_{\mathrm{m}}\right)$ in the middle, and lastly the two objective functions $\left(A_{\Delta P} / A_{\Delta P \max }, A_{\eta}\right)$ on the right. Each dark cyan line represents the corresponding results for one specific individual (CFD results obtained for a specific set of the values of the 8 design parameters). At the end of the optimization, the Paretooptimal set was found to contain five equally optimal (i.e., non-dominated) inducer configurations, which are shown in Figure 9 as thick black lines. It can be seen that these black lines coincide closely for many of the parameters, meaning that "good designs tend to form a family"; however, these optimal designs do show large variations for a few quantities.

To illustrate this point, all black lines correspond to large values of $\beta$ and small values of $C / d$, respectively. This means that it is favorable for both objectives to increase the hub taper angle and to decrease the tip clearance gap. Looking now at the derived variables, the hub diameter $\left(D_{\mathrm{h}}\right)$ should be minimized, while the tip diameter $\left(D_{\mathrm{t}}\right)$ should be maximized to improve both objective functions. That is to say, the radial extension of the blades should be maximized to ensure optimal performance, which seems intuitive. Furthermore, the thick black lines show that the optimal performance is ensured when the blade length $(L)$, the blade sweep angle $(\varphi)$, the blade thickness (both $T_{\mathrm{h}}$ and $T_{\mathrm{t}}$ ) are kept low. A low blade length $L$ and blade chord length $C_{\mathrm{m}}$ as well as a low blade thickness are useful to avoid excess flow blockage, ensuring a smooth flow through the inducer. Unlike the tip clearance gap $(C)$, which should be close to its minimum limit, the blade length and the blade chord length should be low in the provided scales, but at the same time not too low, in order to still provide enough blade length for effective pressure head production. 


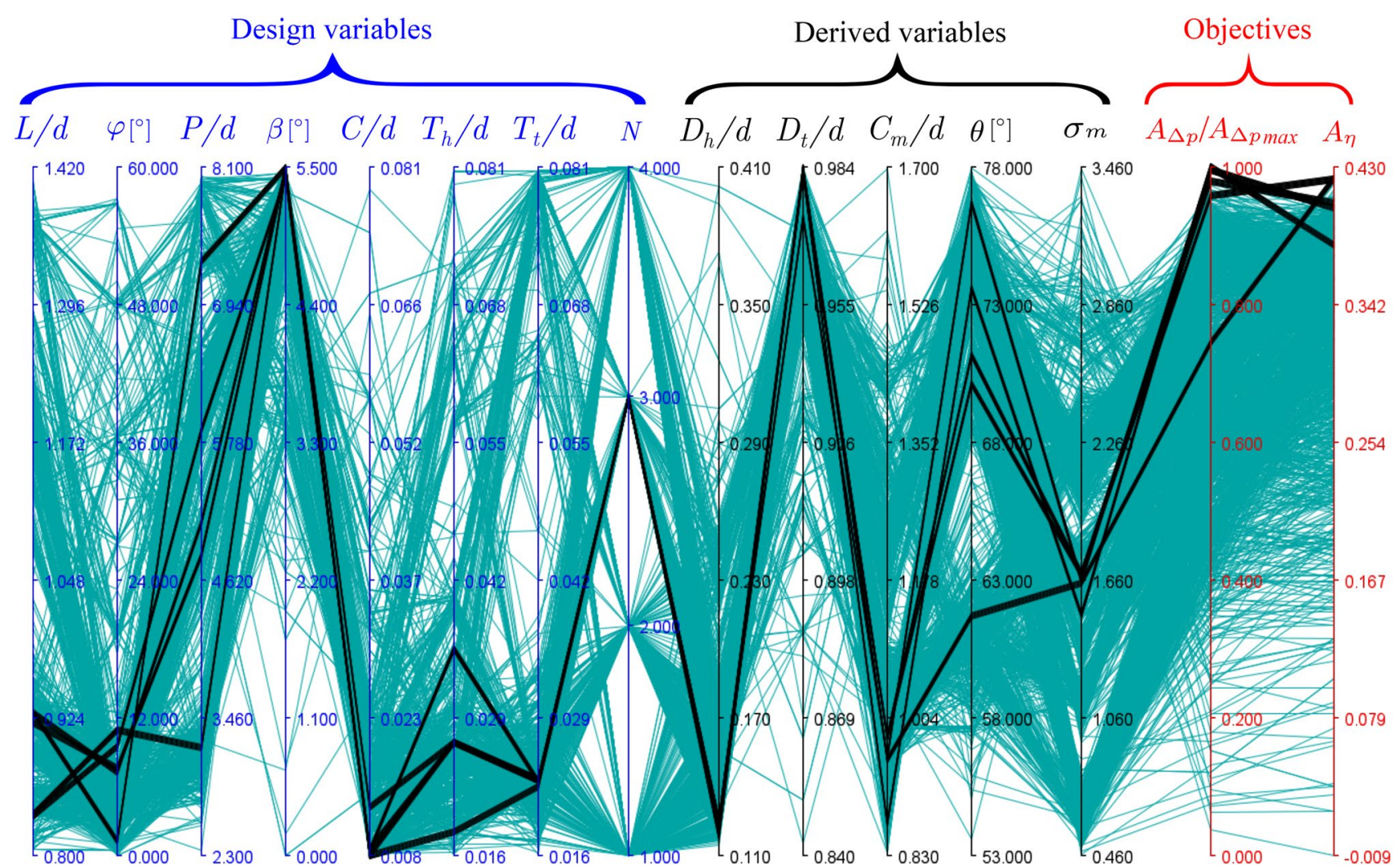

Fig. 9 Parallel coordinates showing the design variables on the left, the derived variables in the middle, and the objectives on the right (each coordinate is shown with its own scale between lower and upper range limits). Each configuration is depicted with a dark cyan line. The members of the Pareto-optimal set (i.e., the best configurations) are marked with thick black lines)
Interestingly, all optimal inducers have $N=3$ blades, which appears to be clearly the most effective solution. Additionally, the solidity range of all optimal designs is limited within $1.5 \leq \sigma_{\mathrm{m}} \leq 1.7$, which is also one of the most important results; this solidity range would be strongly recommended to ensure optimal performance over a wide flow range. This result can be explained by the fact that a low solidity means very short blades, limiting the generated head; on the other hand, the flow is blocked when the solidity is too high, resulting in poor performance. Hence, an intermediate range should indeed be optimal and is quantitatively identified here as $\sigma_{\mathrm{m}} \approx 1.6$. Nonetheless, the optimal configurations are widely spread regarding pitch $(P)$, and accordingly helix angle $\theta$, revealing that high performance can be obtained for inducers with very different values of these parameters. For instance, a high pitch inducer contributes to a higher pressure head and lower efficiency while the opposite is true for a low pitch inducer. Looking finally at the objective functions, it can be seen that changing the geometrical parameters strongly impacts pressure head and/or efficiency. Some designs barely lead to any performance improvement.
The relation between the two objective functions is shown in Fig. 10 in the form of a scatter plot for all designs together with the Pareto set. The horizontal axis represents the normalized integrated pressure head $\left(A_{\Delta P} / A_{\Delta P \max }\right)$ and the vertical axis represents the integrated efficiency $\left(A_{\eta}\right)$. The integrated pressure head has been normalized to its maximum value in the whole analysis to ensure fair comparisons and selections of optimal configurations as discussed later. The Pareto front is shown by thick black circles connected with a solid line, while all other individuals are shown as scattered (gray) crosses. The goal is to reach as far as possible toward the top-right corner of the plot, which would ensure the highest possible efficiency and pressure head simultaneously. As shown in Fig. 10, the Pareto set contains five different optimal inducers. Each optimal individual was assigned with a unique identity number (ID) by sorting them according to increasing pressure head (same as decreasing efficiency). Accordingly, ID 1 has the largest efficiency, while ID 5 possesses the lowest efficiency but the largest pressure head in the Pareto front.

Figure 11 shows the inducer geometries for the five Pareto front individuals. An isometric view, a side view, and a front view of the inducers are shown on the left, middle, 
Fig. 10 Relation between the objective functions for all designs (gray crosses) and the Pareto-optimal set (black circles)

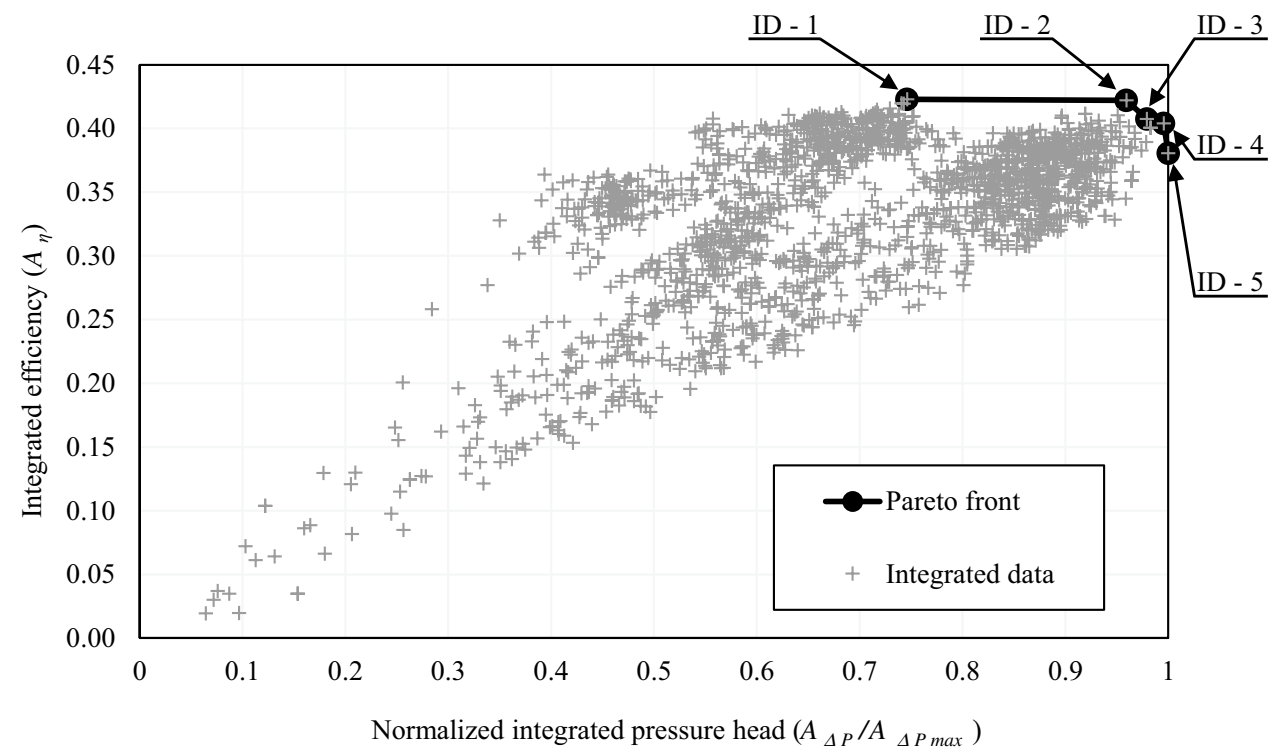

and right columns, respectively. It can be noticed that all the five inducers have generally comparable geometrical features. For instance, they all have-as discussed previously-high hub taper angle, low blade thickness, small axial blade length, which are necessary to ensure high performance. Nonetheless, they have different blade pitch values. That is why the blades of inducer 1 appear more twisted than the other inducers (low pitch). Note that the pitch value increases monotonically from ID 1 to ID 5, leading to a gradual increase in the integrated pressure head and a decrease in efficiency, respectively. The exact values of all variables of each optimal individual are listed in Table 2. The table also shows the upper and lower limits of all variables in the last column for the Pareto set, which can be used as recommended ranges for any optimal design.

Figure $12 \mathrm{a}$ and $\mathrm{b}$ show the normalized pressure head $\left(\Delta P / \Delta P_{\max }\right)$ and the efficiency $(\eta)$ curves, respectively, for the five Pareto front individuals as a function of the normalized flow rate $\left(Q / Q_{\text {opt }}\right)$. Our previous studies showed a significant negative inducer performance at overload conditions for the unoptimized original inducer (see Fig. 2) due to the occurrence of strong flow separation at the leading edge of the blades, which leads to the formation of large axially propagating vortices across the inducer (Mansour et al. 2019, 2020a, b) (see also Fig. 18). It is important to note that the entire performance curves of the optimized inducers are now positive everywhere, showing also high performance near the optimal flow, i.e., matching the whole pump flow range. In other words, the maximum efficiency points of all optimized inducers are mostly occurring at $Q / Q_{\text {opt }}=1$, which is the maximum efficiency point of the pump.

Inducer 1 , although having a decent performance and highest efficiency at part-load $\left(Q / Q_{\mathrm{opt}}=0.8\right)$ conditions, is unable to maintain high pressure head at optimal
$\left(Q / Q_{\mathrm{opt}}=1.0\right)$ and overload $\left(Q / Q_{\mathrm{opt}}=1.5\right)$ conditions as seen in Fig. 12a. The reason would be that this inducer has a relatively small pitch and a high blade sweep angle as compared to other individuals in the Pareto set. These conditions might block the fluid motion at high flow but appears to be very useful at low-flow conditions. The other four individuals are able to maintain an effective pressure head at all flow conditions.

\subsection{Analysis for part-load $\left(Q / Q_{\text {opt }}=0.8\right)$, optimal $\left(Q / Q_{\text {opt }}=1.0\right)$ and overload conditions $\left(Q / Q_{\text {opt }}=1.5\right)$}

This section extends the study by further understanding the effect of various geometrical parameters on the inducer performance at each considered flow rate individually, i.e., part-load, optimal, and overload conditions. Thus, in the following subsections, the (non-integrated) normalized pressure head and the normalized efficiency have been used as the objective functions separately at part-load, optimal, or overload conditions to determine optimal inducer designs for each load condition, individually.

\subsubsection{Part-load flow conditions $\left(Q / Q_{\text {opt }}=0.8\right)$}

At part-load conditions, seven optimal inducer configurations were found from the present analysis (given ID 6-12). Table 3 lists the values of the design variables, derived variables, and the objective functions for all optimal individual at part-load conditions $\left(Q / Q_{\mathrm{opt}}=0.8\right)$, together with the corresponding optimal ranges of all variables. Most geometrical characteristics concerning the optimal designs at part-load remain the same as for the optimal set of the integrated data discussed previously. However, optimal performance can 


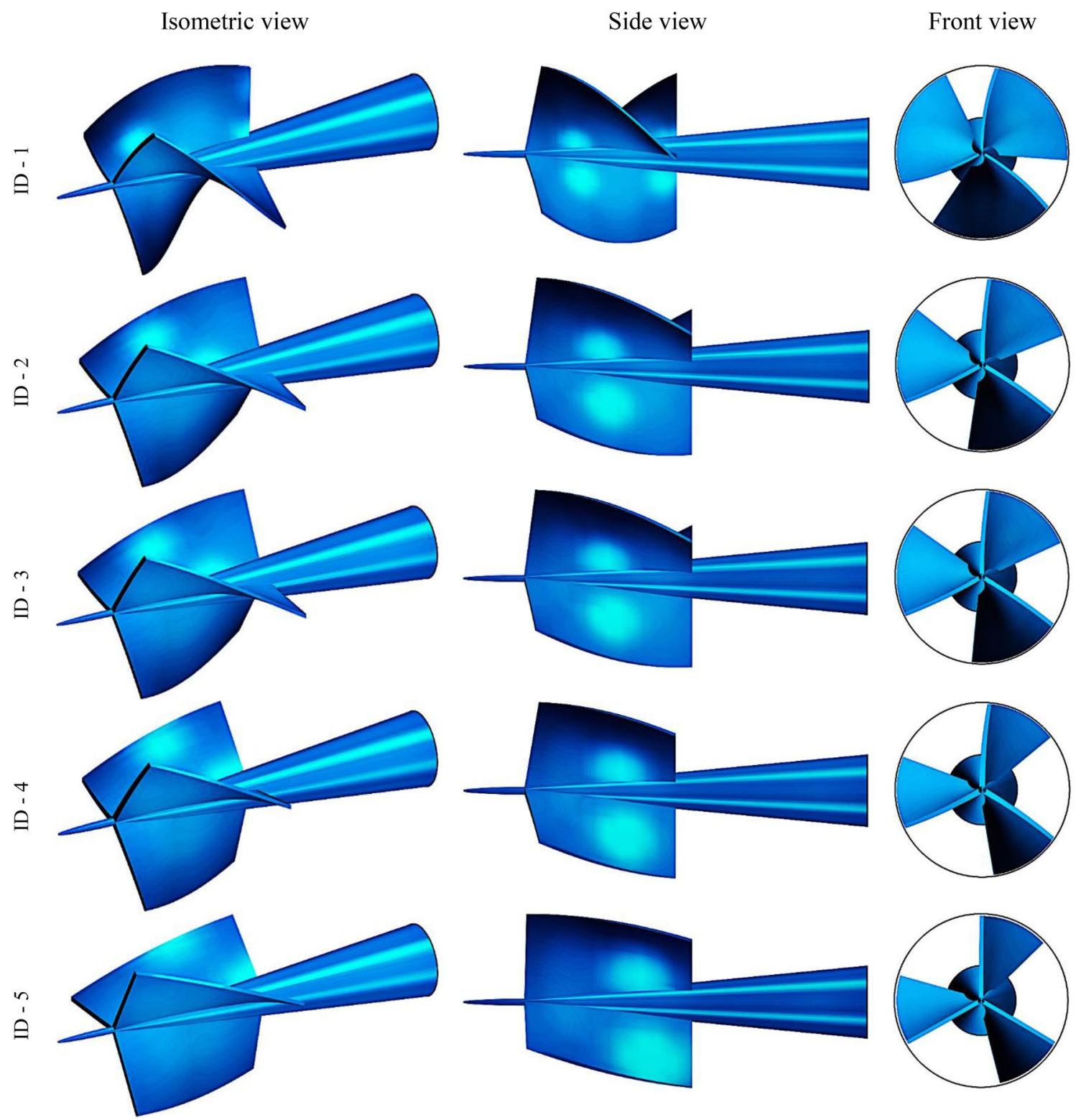

Fig. 11 Geometrical views of the optimal designs - isometric (left), side (middle), and front (right) views

be obtained by inducers having a various number of blades ( $N=2,3$, or 4) at part-load conditions, unlike the integrated data. Furthermore, the optimal solidity range is now wider than the previous case, revealing that the solidity becomes not very critical since the flow is slow at these conditions and cannot be easily blocked by the inducer.

Figure $13 \mathrm{a}$ and $\mathrm{b}$ show the normalized pressure head $\left(\Delta P / \Delta P_{\max }\right)$ and the efficiency $(\eta)$ curves for the seven Pareto front individuals as a function of the normalized flow rate $\left(Q / Q_{\text {opt }}\right)$. As can be seen, these optimal inducers show a peak performance at part-load flow, which decreases monotonically with the increase of the flow.
Further, this analysis confirms a concurrent behavior of the objective functions. Here a high efficiency is achieved at part-load conditions by low pitch, a low number of blades, and a relatively higher sweep angle. These conditions are all satisfied with ID 6. However, inducer 6 fails to compete with other Pareto front individuals concerning the pressure head, where it shows a consistent lower performance at all flow conditions. On the other hand, to achieve a high pressure head at part-load conditions, high pitch, a high number of blades, and a low sweep angle are recommended (ID 11 and ID 12). 
Table 2 Variable values for the Pareto set of the integrated data

\begin{tabular}{|c|c|c|c|c|c|c|c|}
\hline \multirow[t]{2}{*}{ Optimal designs } & & \multicolumn{5}{|c|}{ Inducer ID } & \multirow[t]{2}{*}{ All optimal designs } \\
\hline & & 1 & 2 & 3 & 4 & 5 & \\
\hline \multirow[t]{8}{*}{ Design variables } & $L / d$ & 0.838 & 0.920 & 0.929 & 0.836 & 0.929 & $0.836 \leq L / d \leq 0.929$ \\
\hline & $\varphi\left[^{\circ}\right]$ & 10.929 & 7.630 & 7.301 & 9.496 & 1.341 & $1.341 \leq \varphi \leq 10.929$ \\
\hline & $P / d$ & 3.217 & 4.829 & 5.109 & 5.891 & 7.305 & $3.217 \leq P / d \leq 7.305$ \\
\hline & $\beta\left[^{\circ}\right]$ & 5.463 & 5.483 & 5.495 & 5.424 & 5.495 & $5.424 \leq \beta \leq 5.495$ \\
\hline & $C / d$ & 0.0081 & 0.0081 & 0.0088 & 0.0086 & 0.0132 & $0.0081 \leq C / d \leq 0.0132$ \\
\hline & $T_{\mathrm{h}} / d$ & 0.0182 & 0.0267 & 0.0268 & 0.0356 & 0.0266 & $0.0182 \leq T_{\mathrm{h}} / d \leq 0.0356$ \\
\hline & $T_{\mathrm{t}} / d$ & 0.0226 & 0.0232 & 0.0231 & 0.0230 & 0.0231 & $0.0226 \leq T_{\mathrm{t}} / d \leq 0.0232$ \\
\hline & $N$ & 3 & 3 & 3 & 3 & 3 & $N=3$ \\
\hline \multirow[t]{5}{*}{ Derived variables } & $D_{\mathrm{h}} / d$ & 0.118 & 0.125 & 0.126 & 0.120 & 0.126 & $0.118 \leq D_{\mathrm{h}} / d \leq 0.126$ \\
\hline & $D_{\mathrm{t}} / d$ & 0.984 & 0.984 & 0.982 & 0.983 & 0.974 & $0.974 \leq D_{\mathrm{t}} / d \leq 0.984$ \\
\hline & $C_{\mathrm{m}} / d$ & 0.951 & 0.878 & 0.981 & 0.872 & 0.955 & $0.872 \leq C_{\mathrm{m}} / d \leq 0.981$ \\
\hline & $\theta\left[^{\circ}\right]$ & 61.71 & 70.16 & 71.19 & 73.61 & 76.70 & $61.71 \leq \theta \leq 76.70$ \\
\hline & $\sigma_{\mathrm{m}}$ & 1.648 & 1.684 & 1.691 & 1.509 & 1.659 & $1.509 \leq \sigma_{\mathrm{m}} \leq 1.691$ \\
\hline \multirow[t]{2}{*}{ Objectives } & $A_{\Delta P} / A_{\Delta P \max }$ & 0.746 & 0.959 & 0.979 & 0.996 & 1.000 & $0.746 \leq A_{\Delta P} / A_{\Delta P \max } \leq 1$ \\
\hline & $A_{\eta}$ & 0.423 & 0.422 & 0.407 & 0.404 & 0.381 & $0.381 \leq A_{\eta} \leq 0.423$ \\
\hline
\end{tabular}

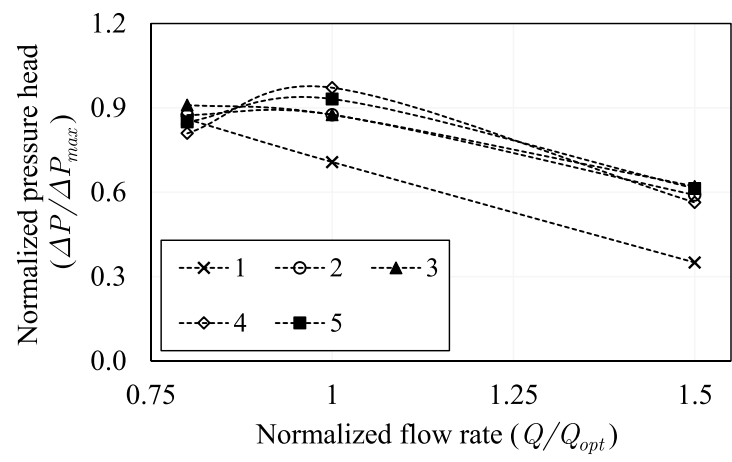

(a) Normalized pressure head.

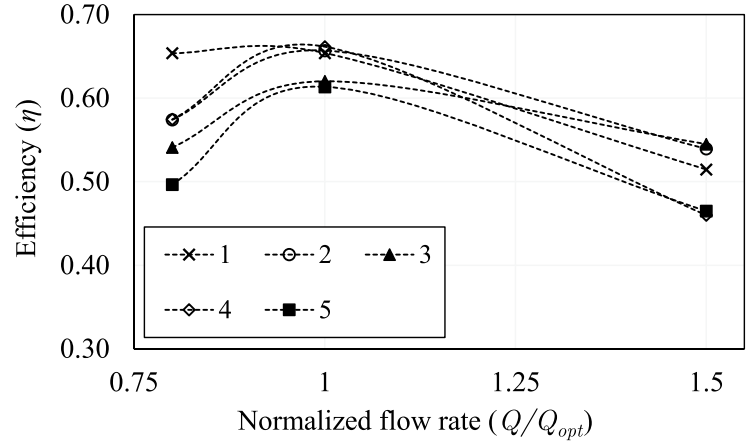

(b) Efficiency.

Fig. 12 Performance curves for Pareto set of the integrated data

\subsubsection{Optimal flow conditions $\left(Q / Q_{o p t}=1.0\right)$}

At optimal flow conditions, there exist two optimal inducers (ID 4 and ID 13) as listed in Table 4. Again, a number of blades of 3 is found most suitable at optimal flow, and the solidity range is once again limited to a narrow range $\left(1.5 \leq \sigma_{\mathrm{m}} \leq 1.8\right)$ for ensuring best performance. All other geometrical parameters are very comparable with the previous optimal cases.

Figure 14a and $\mathrm{b}$ shows the normalized pressure head $\left(\Delta P / \Delta P_{\max }\right)$ and the efficiency $(\eta)$ curves for the two Pareto individuals of the optimal flow as a function of the normalized flow rate $\left(Q / Q_{\mathrm{opt}}\right)$. Although ID 13 provides slightly better efficiency at part-load and optimal conditions compared to that of ID 4 , it has a consistently lower pressure head, especially at optimal and overload conditions. Therefore, inducer 4 is normally preferred to inducer
13 to ensure effective performance not only for optimal flow conditions but also for a wide range of flow rates.

\subsubsection{Overload flow conditions $\left(Q / Q_{\mathrm{opt}}=1.5\right)$}

The Pareto set at overload flow conditions contains 5 optimal inducer configurations (ID 3 and ID 14 to 17). The corresponding variable values of the Pareto set are listed in Table 5. Accordingly, 17 distinct optimal inducers could be identified in the whole study at different flow conditions. The optimal inducers at overload conditions show - like that at optimal conditions - low sweep angle, high hub taper angle, and a high number of blades. Additionally, the blade thickness and the tip clearance gap are likewise recommended to be kept low. Nonetheless, the pitch range is noticeably higher compared to all previous optimal sets to avoid blocking the flow at such high flow conditions. 
Table 3 Variable values for the Pareto set of the part-load flow

\begin{tabular}{|c|c|c|c|c|c|c|c|c|c|}
\hline \multicolumn{2}{|c|}{ Pareto front inducer designs } & \multicolumn{7}{|c|}{ Inducer ID } & \multirow[t]{2}{*}{ Pareto-optimal set } \\
\hline & & 6 & 7 & 8 & 9 & 10 & 11 & 12 & \\
\hline \multirow[t]{8}{*}{ Design variables } & $L / d$ & 0.838 & 0.839 & 0.929 & 0.858 & 0.901 & 0.929 & 0.942 & $0.838 \leq L / d \leq 0.942$ \\
\hline & $\varphi\left[^{\circ}\right]$ & 10.805 & 3.907 & 7.311 & 7.567 & 10.219 & 2.581 & 0.576 & $0.576 \leq \varphi \leq 10.805$ \\
\hline & $P / d$ & 2.751 & 4.909 & 3.359 & 4.819 & 4.827 & 4.233 & 6.217 & $2.751 \leq P / d \leq 6.217$ \\
\hline & $\beta\left[^{\circ}\right]$ & 5.182 & 5.137 & 5.495 & 5.482 & 5.482 & 4.438 & 5.051 & $4.438 \leq \beta \leq 5.495$ \\
\hline & $C / d$ & 0.0087 & 0.0133 & 0.0087 & 0.0083 & 0.0083 & 0.0088 & 0.0093 & $0.0083 \leq C / d \leq 0.0133$ \\
\hline & $T_{\mathrm{h}} / d$ & 0.0227 & 0.0263 & 0.0268 & 0.0202 & 0.0201 & 0.0268 & 0.0247 & $0.0201 \leq T_{\mathrm{h}} / d \leq 0.0268$ \\
\hline & $T_{\mathrm{t}} / d$ & 0.0226 & 0.0225 & 0.0231 & 0.0232 & 0.0307 & 0.0226 & 0.0226 & $0.0225 \leq T_{\mathrm{t}} / d \leq 0.0307$ \\
\hline & $N$ & 2 & 4 & 4 & 3 & 4 & 4 & 3 & $2 \leq N \leq 4$ \\
\hline \multirow[t]{5}{*}{ Derived variables } & $D_{\mathrm{h}} / d$ & 0.133 & 0.136 & 0.126 & 0.119 & 0.124 & 0.179 & 0.149 & $0.119 \leq D_{\mathrm{h}} / d \leq 0.179$ \\
\hline & $D_{\mathrm{t}} / d$ & 0.983 & 0.973 & 0.983 & 0.983 & 0.983 & 0.982 & 0.981 & $0.973 \leq D_{\mathrm{t}} / d \leq 0.983$ \\
\hline & $C_{\mathrm{m}} / d$ & 0.993 & 0.900 & 1.046 & 0.912 & 0.957 & 1.011 & 0.980 & $0.900 \leq C_{\mathrm{m}} / d \leq 1.046$ \\
\hline & $\theta\left[^{\circ}\right]$ & 57.50 & 70.47 & 62.61 & 70.23 & 70.19 & 66.69 & 74.06 & $57.50 \leq \theta \leq 74.06$ \\
\hline & $\sigma_{\mathrm{m}}$ & 1.134 & 2.043 & 2.405 & 1.580 & 2.202 & 2.218 & 1.656 & $1.134 \leq \sigma_{\mathrm{m}} \leq 2.405$ \\
\hline \multirow[t]{2}{*}{ Objectives } & $\Delta P / \Delta P_{\max }$ & 0.729 & 0.889 & 0.944 & 0.954 & 0.968 & 0.983 & 1.000 & $0.729 \leq \Delta P / \Delta P_{\max } \leq 1.000$ \\
\hline & $\eta_{\text {part }}$ & 0.660 & 0.657 & 0.636 & 0.625 & 0.611 & 0.607 & 0.592 & $0.592 \leq \eta_{\text {part }} \leq 0.660$ \\
\hline
\end{tabular}

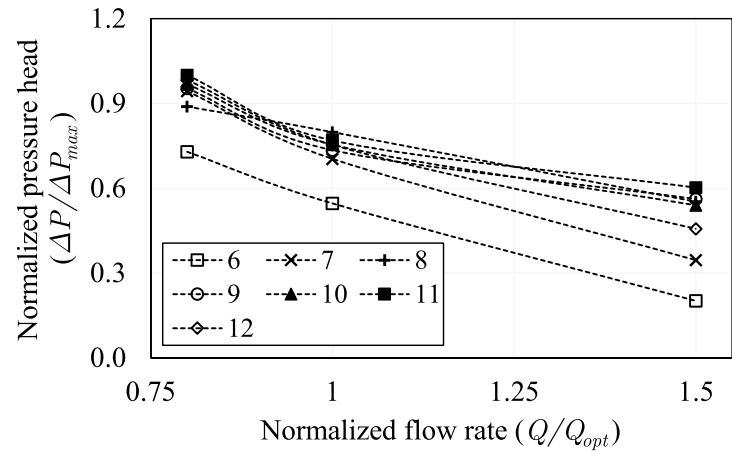

(a) Normalized pressure head

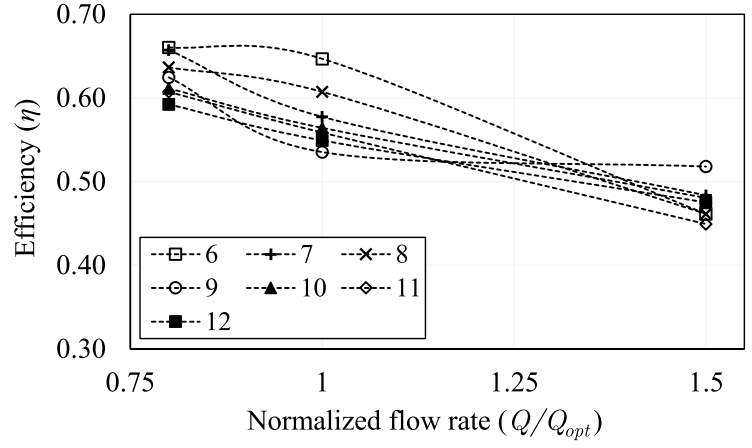

(b) Efficiency.

Fig. 13 Performance curves for the Pareto set of the part-load flow

Figure $15 \mathrm{a}$ and $\mathrm{b}$ show the normalized pressure head $\left(\Delta P / \Delta P_{\max }\right)$ and the efficiency $(\eta)$ curves for the Pareto set of the overload flow as a function of the normalized flow rate $\left(Q / Q_{\text {opt }}\right)$. One important note here is that the performance curves of these inducers are mostly flat and do not drop significantly with the increase of the flow rate. Accordingly, they all have an optimal performance at overload flow. Such designs are particularly important for applications needing an almost constant performance for a wide range of flow rates. It can be seen that ID 17 generates the maximum pressure head at overload conditions. However, it has the lowest efficiency compared to all other inducers in the Pareto front; its peak efficiency would occur far in the overload regime. The reason is that it has a relatively long axial blade length and high pitch. Note again in Table 5 that the overload efficiency decreases with the increase in pitch, while the generated pressure head increases. Final suggestions for best inducer geometries are discussed in the next section.

\subsection{Selection of optimal inducers}

Considering all the optimal Pareto front inducer configurations as discussed up to now, an optimal geometry can already be chosen based on specific objectives, i.e., whether head or efficiency is of main concern, and which flow rate is targeted. In the present section, the previous analysis is extended by suggesting a way in which an optimal inducer configuration could be chosen. The selection is done based on the inducer having minimum Euclidean distance to the "Utopia" point as shown in Fig. 16, where both objective functions would be ideally maximum. This is achieved at $A_{\Delta P} / A_{\Delta P \max }=1.0$ and $A_{\eta} / A_{\eta \max }=1$. The theoretical maximum of the integrated efficiency $\left(A_{\eta \max }\right)$ can be determined 
Table 4 Variable values for the Pareto set of the optimal flow

\begin{tabular}{|c|c|c|c|c|}
\hline \multicolumn{2}{|c|}{ Pareto front inducer designs } & \multicolumn{2}{|c|}{ Inducer ID } & \multirow[t]{2}{*}{ Pareto-optimal set } \\
\hline & & 13 & 4 & \\
\hline \multirow[t]{8}{*}{ Design variables } & $L / d$ & 0.858 & 0.836 & $0.836 \leq L / d \leq 0.858$ \\
\hline & $\varphi\left[^{\circ}\right]$ & 3.954 & 9.496 & $3.954 \leq \varphi \leq 9.496$ \\
\hline & $P / d$ & 2.592 & 5.891 & $2.592 \leq P / d \leq 5.891$ \\
\hline & $\beta\left[^{\circ}\right]$ & 5.482 & 5.424 & $5.424 \leq \beta \leq 5.482$ \\
\hline & $C / d$ & 0.0083 & 0.0086 & $0.0083 \leq C / d \leq 0.0086$ \\
\hline & $T_{\mathrm{h}} / d$ & 0.0182 & 0.0356 & $0.0182 \leq T_{\mathrm{h}} / d \leq 0.0356$ \\
\hline & $T_{\mathrm{t}} / d$ & 0.0265 & 0.0230 & $0.0230 \leq T_{\mathrm{t}} / d \leq 0.0265$ \\
\hline & $N$ & 3 & 3 & $N=3$ \\
\hline \multirow[t]{5}{*}{ Derived variables } & $D_{\mathrm{h}} / d$ & 0.119 & 0.120 & $0.119 \leq D_{\mathrm{h}} / d \leq 0.120$ \\
\hline & $D_{\mathrm{t}} / d$ & 0.984 & 0.983 & $0.973 \leq D_{\mathrm{t}} / d \leq 0.984$ \\
\hline & $C_{\mathrm{m}} / d$ & 1.032 & 0.872 & $0.872 \leq C_{\mathrm{m}} / d \leq 1.032$ \\
\hline & $\theta\left[^{\circ}\right]$ & 56.24 & 73.61 & $56.24 \leq \theta \leq 73.61$ \\
\hline & $\sigma_{\mathrm{m}}$ & 1.788 & 1.509 & $1.509 \leq \sigma_{\mathrm{m}} \leq 1.788$ \\
\hline \multirow[t]{2}{*}{ Objectives } & $\Delta P / \Delta P_{\max }$ & 0.579 & 0.972 & $0.579 \leq \Delta P / \Delta P_{\max } \leq 0.972$ \\
\hline & $\eta_{\mathrm{opt}}$ & 0.672 & 0.662 & $0.662 \leq \eta_{\mathrm{opt}} \leq 0.672$ \\
\hline
\end{tabular}

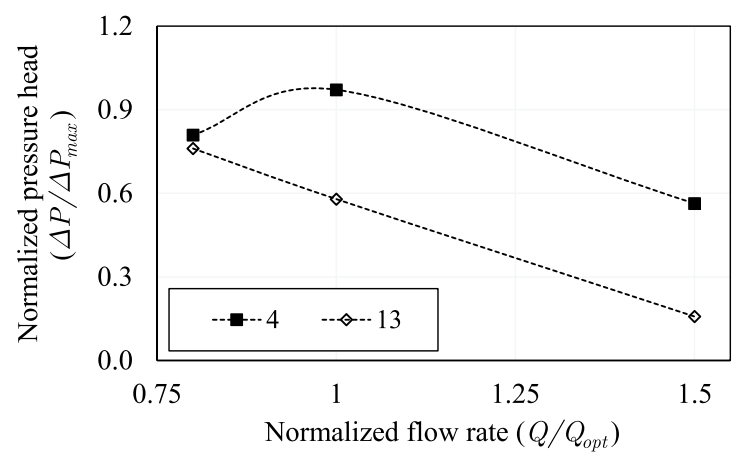

(a) Normalized pressure head.

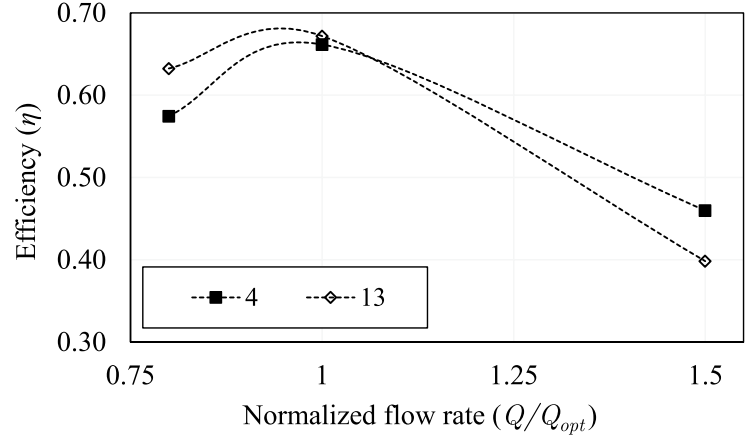

(b) Efficiency.

Fig. 14 Performance curves for the Pareto set of the optimal flow

Table 5 Variable values for the Pareto set of the overload flow

\begin{tabular}{|c|c|c|c|c|c|c|c|}
\hline \multicolumn{2}{|c|}{ Pareto front inducer designs } & \multicolumn{5}{|c|}{ Inducer ID } & \multirow[t]{2}{*}{ Pareto-optimal set } \\
\hline & & 14 & 3 & 15 & 16 & 17 & \\
\hline \multirow[t]{8}{*}{ Design variables } & $L / d$ & 0.901 & 0.929 & 0.893 & 0.943 & 1.133 & $0.893 \leq L / d \leq 1.133$ \\
\hline & $\varphi\left[^{\circ}\right]$ & 7.658 & 7.301 & 0.606 & 0.548 & 3.044 & $0.548 \leq \varphi \leq 7.658$ \\
\hline & $P / d$ & 4.819 & 5.109 & 5.841 & 6.188 & 7.953 & $4.819 \leq P / d \leq 7.953$ \\
\hline & $\beta\left[^{\circ}\right]$ & 5.497 & 5.495 & 5.413 & 5.293 & 5.049 & $5.049 \leq \beta \leq 5.497$ \\
\hline & $C / d$ & 0.0088 & 0.0088 & 0.0141 & 0.0093 & 0.0199 & $0.0088 \leq C / d \leq 0.0199$ \\
\hline & $T_{\mathrm{h}} / d$ & 0.0192 & 0.0268 & 0.0201 & 0.0191 & 0.0263 & $0.0191 \leq T_{\mathrm{h}} / d \leq 0.0268$ \\
\hline & $T_{\mathrm{t}} / d$ & 0.0171 & 0.0231 & 0.0232 & 0.0226 & 0.0240 & $0.0171 \leq T_{\mathrm{t}} / d \leq 0.0240$ \\
\hline & $N$ & 3 & 3 & 4 & 3 & 3 & $3 \leq N \leq 4$ \\
\hline \multirow[t]{5}{*}{ Derived variables } & $D_{\mathrm{h}} / d$ & 0.123 & 0.126 & 0.126 & 0.137 & 0.166 & $0.123 \leq D_{\mathrm{h}} / d \leq 0.166$ \\
\hline & $D_{\mathrm{t}} / d$ & 0.982 & 0.982 & 0.972 & 0.981 & 0.960 & $0.960 \leq D_{\mathrm{t}} / d \leq 0.982$ \\
\hline & $C_{\mathrm{m}} / d$ & 0.957 & 0.981 & 0.931 & 0.980 & 1.160 & $0.931 \leq C_{\mathrm{m}} / d \leq 1.160$ \\
\hline & $\theta\left[^{\circ}\right]$ & 70.19 & 71.19 & 73.55 & 74.15 & 77.46 & $70.19 \leq \theta \leq 77.46$ \\
\hline & $\sigma_{\mathrm{m}}$ & 1.654 & 1.691 & 2.159 & 1.674 & 1.967 & $1.654 \leq \sigma_{\mathrm{m}} \leq 2.159$ \\
\hline \multirow[t]{2}{*}{ Objectives } & $\Delta P / \Delta P_{\max }$ & 0.608 & 0.621 & 0.668 & 0.669 & 0.692 & $0.608 \leq \Delta P / \Delta P_{\text {max }} \leq 0.692$ \\
\hline & $\eta_{\text {over }}$ & 0.556 & 0.545 & 0.538 & 0.529 & 0.509 & $0.509 \leq \eta_{\text {over }} \leq 0.556$ \\
\hline
\end{tabular}




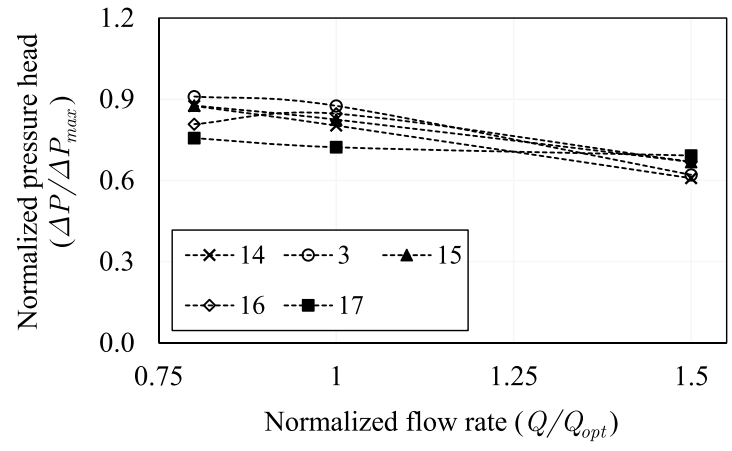

(a) Normalized pressure head.

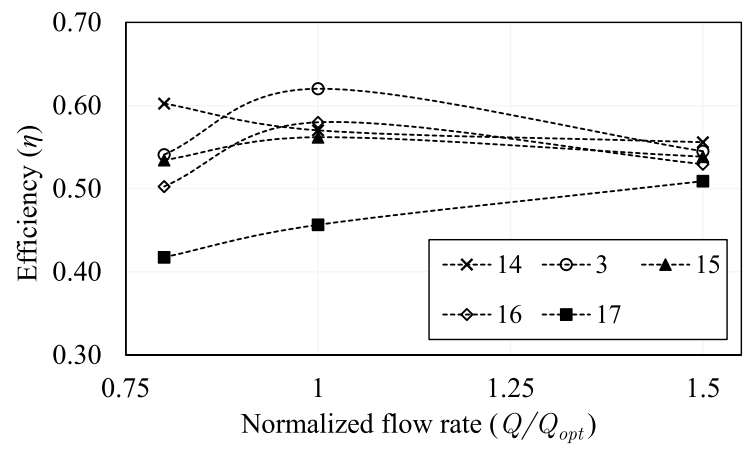

(b) Efficiency.

Fig. 15 Performance curves for the Pareto set of the overload flow

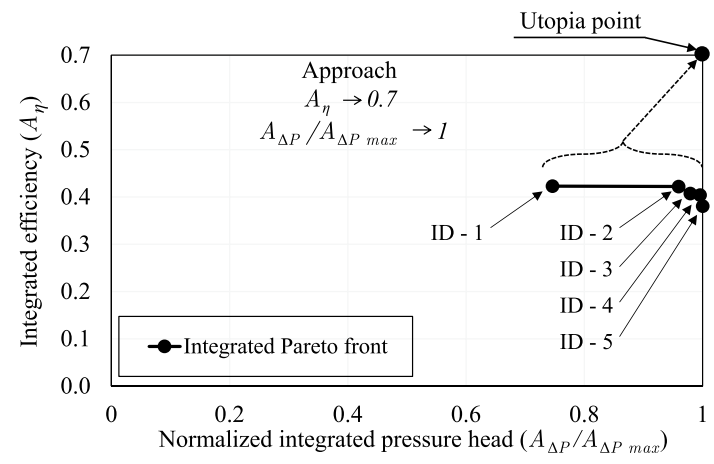

(a) Integrated Pareto front along with the Utopia point

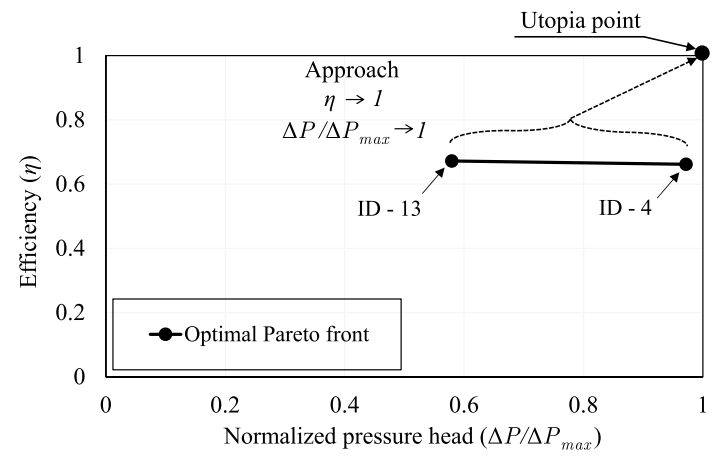

(c) Optimal Pareto front along with the Utopia point

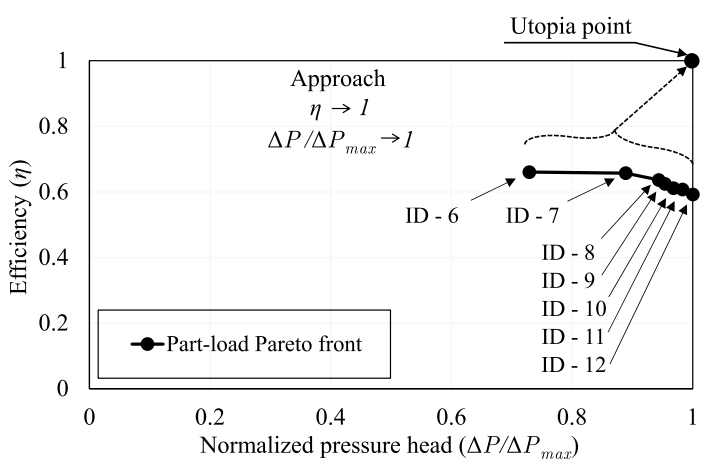

(b) Part-load Pareto front along with the Utopia point

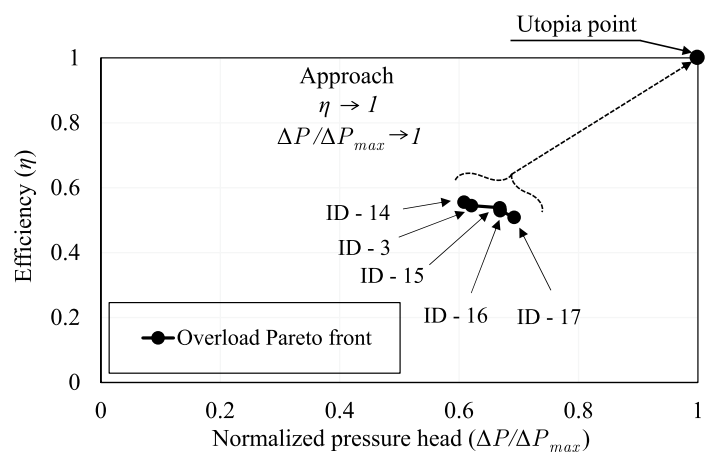

(d) Overload Pareto front along with the Utopia point

Fig. 16 Pareto front obtained from optimization along with the desired Utopia point

from Eq. (16) by setting $\eta_{\text {part }}=\eta_{\text {opt }}=\eta_{\text {over }}=1.0$, leading to $A_{\text {nmax }}=0.7$. However, the maximum possible objective functions are achieved separately for part-load, optimal, or overload conditions at $\Delta P / \Delta P_{\max }=1$ and $\eta=1.0$. Figure 16 shows the four Pareto fronts obtained in the present study at different conditions and each corresponding Utopia point. The distance from the data point of each inducer on the plot of the objective functions to the Utopia point is calculated based on the Euclidean norm for all cases and listed in Table 6 . The inducers are sorted by increasing distance to the Utopia point. Accordingly, inducer ID - 2, 7, 4, and 15 (shown in bold in Table 6) have minimum distances to the Utopia points and are thus recommended for overall integrated, part-load, optimal, and overload working conditions, respectively.

Finally, a comparison between the four selected optimal configurations and the original unoptimized inducer geometry is discussed. Figure $17 \mathrm{a}$ and $\mathrm{b}$ show the normalized pressure head $\left(\Delta P / \Delta P_{\max }\right)$ and the efficiency $(\eta)$ curves for the four selected individuals along with the original 
unoptimized inducer used in our previous studies (Mansour et al. 2018b, 2019, 2020a, b; Parikh et al. 2020) as a function of the normalized flow rate $\left(Q / Q_{\mathrm{opt}}\right)$. The original inducer is added to the comparison here to show how far the optimized inducers can improve the performance beyond this available, classically designed, and frequently employed inducer. As seen in Fig. 17, no matter the flow conditions, the four selected individuals generate significantly higher pressure heads than the previous unoptimized inducer. Simultaneously, a higher peak efficiency point with a much larger working flow range is ensured with the optimized inducers. As shown the unoptimized inducer failed to generate any reasonable performance for overload conditions due to the big flow separation and the strong axial vortices. The overload performance is now very efficiently improved when using any of the recommended optimal inducers.

Figure 18 compares the flow patterns for radial sections at two $x$ distances from the domain inlet, i.e., within $(x=3.54 d)$, and downstream $(x=4.19 d)$ of the blades for the four recommended optimized inducers and the unoptimized inducer at $Q / Q_{\text {opt }}=1.5$. The five columns (left to right) represent the five inducers (ID - 2, 7, 4, 15, and the unoptimized inducer), while the two rows show two radial sections at different positions (at mid-length of the blades and downstream of the inducer blades). It is clearly seen that all the optimized inducers show only weak, sometimes barely visible vortices, while strong vortical structures appear near all blades of the unoptimized inducer. It is also worthwhile to note that using an inducer with 4 blades (ID 7 and 15) can damp the vortex generation downstream of the blades. The reason is that the flow is more streamlined and better guided with an increase in the number of blades. In addition to the previous results, this visualization confirms that inducer 15 is the best individual at overload conditions.

\section{Conclusions}

A CFD-based multi-objective optimization was carried out for pump inducers to maximize simultaneously pressure head and efficiency. To ensure high performance for a wide flow range, the areas under the pressure and efficiency curves are considered as the two objective functions to be maximized. This is achieved by integrating the pressure and efficiency curves at three different normalized flow rates, i.e., $Q / Q_{\text {opt }}=0.8,1.0$, and 1.5. All important geometrical parameters have been varied within wide ranges; axial blade length, blade sweep angle, blade pitch, hub taper angle, tip clearance gap, blade thickness at the hub, blade thickness at the tip, and the number of blades. The optimization process was done using an inhouse optimization code with the efficient, multi-objective 


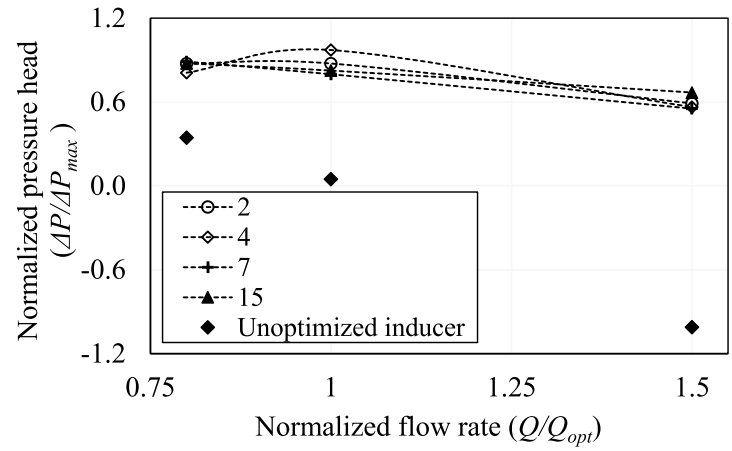

(a) Normalized pressure head.

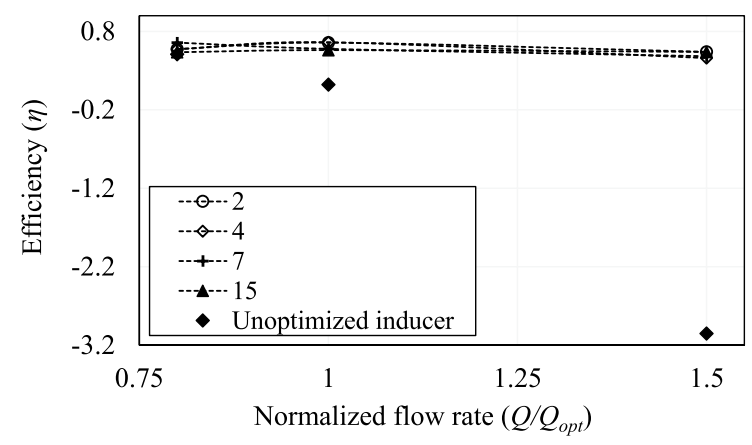

(b) Efficiency.

Fig. 17 Performance curve comparison for the selected optimal individuals with the original unoptimized inducer

ID - 2
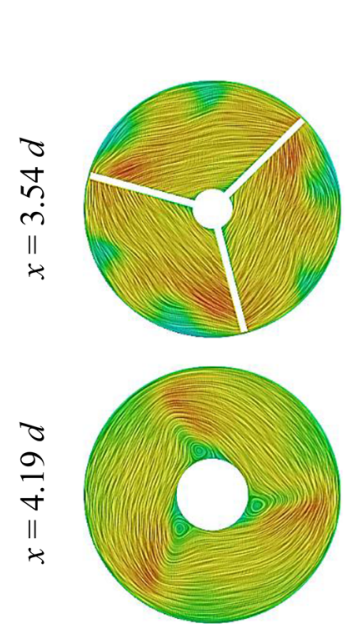

ID - 7
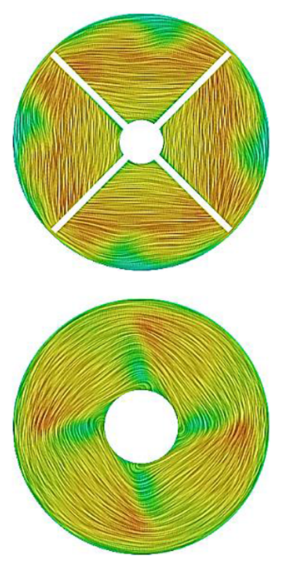

0.00
ID - 4
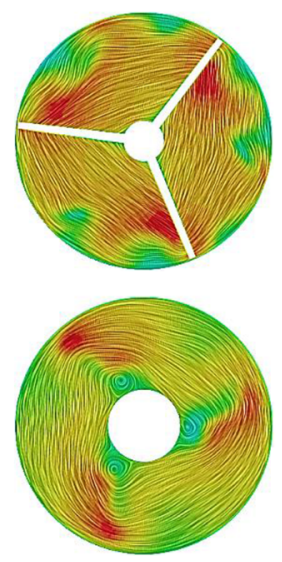

Velocity: Magnitude $(\mathrm{m} / \mathrm{s})$
ID - 15
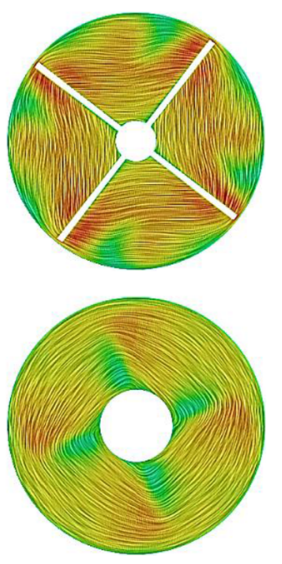

3.60
Unoptimized Inducer
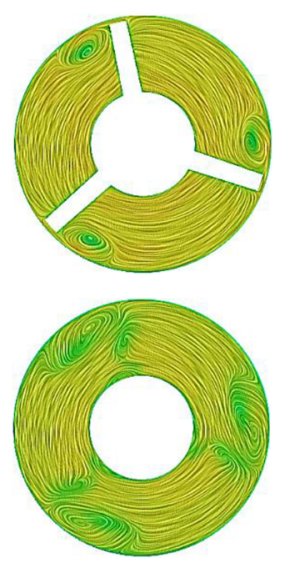

4.50

Fig. 18 Velocity profiles for radial sections at two different $x$ distances from the simulation domain inlet, i.e., within $(x=3.54 d)$, and downstream $(x=4.19 d)$ of the blades at overload conditions $\left(Q / Q_{\text {opt }}=1.5\right)$

global optimization method NSGA-II. A total of 37 generations involving 5178 different CFD simulations have been needed for the optimization process. In the end, five optimal inducer configurations are obtained from the integrated data. Further, a single overall optimal design is proposed based on the integrated data (ID - 2 from Fig. 11) together with three other optimal inducer configurations at each considered flow condition. Analyzing all results, it is seen that a high hub taper angle with 3 blades should be used to maximize inducer performance; additionally, blade length, blade sweep angle, tip clearance gap, and blade thickness should be kept low.
Acknowledgements This work is part of a project funded by VDMA (Verband Deutscher Maschinen- und Anlagenbau) and BMWi (Bundesministeriums für Wirtschaft und Energie) under number IGF 20638.

Funding Open Access funding enabled and organized by Projekt DEAL.

\section{Declarations}

Conflict of Interest The authors declare that they have no conflict of interest.

Replication of results The simulation data for the replication of results can be provided on request. 
Open Access This article is licensed under a Creative Commons Attribution 4.0 International License, which permits use, sharing, adaptation, distribution and reproduction in any medium or format, as long as you give appropriate credit to the original author(s) and the source, provide a link to the Creative Commons licence, and indicate if changes were made. The images or other third party material in this article are included in the article's Creative Commons licence, unless indicated otherwise in a credit line to the material. If material is not included in the article's Creative Commons licence and your intended use is not permitted by statutory regulation or exceeds the permitted use, you will need to obtain permission directly from the copyright holder. To view a copy of this licence, visit http://creativecommons.org/licenses/by/4.0/.

\section{References}

Amoresano A, Langella G, Niola V, Quaremba G (2014) Advanced image analysis of two-phase flow inside a centrifugal pump. Adv Mech Eng 6:1-11

Bakir F, Rey R, Gerber A, Belamri T, Hutchinson B (2004) Numerical and experimental investigations of the cavitating behavior of an inducer. Int J Rotat Mach 10(1):15-25

Campo A, Chisely EA (2010) Experimental characterization of twophase flow centrifugal pumps. In: ASME 2010 Power Conf., American Society of Mechanical Engineers, 2010, 803-816

Campos-Amezcua R, Khelladi S, Mazur-Czerwiec Z, Bakir F, CamposAmezcua A, Rey R (2013) Numerical and experimental study of cavitating flow through an axial inducer considering tip clearance. Proc. IMechE Part A 227(8):858-868

Cappellino CA, Roll DR, Wilson G (1992) Design Considerations and application guidelines for pumping liquids with entrained gas using open impeller centrifugal pumps. In: Proc. 9th Int. Pump Users Symp., Turbomachinery Laboratories, Department of Mechanical Engineering, Texas A\&M University, 262-264

Caridad J, Kenyery F (2004) CFD analysis of electric submersible pumps (ESP) handling two-phase mixtures. J Energy Resour Technol 126(2):99-104

Caridad J, Asuaje M, Kenyery F, Tremante A, Aguillón O (2008) Characterization of a centrifugal pump impeller under two-phase flow conditions. J Pet Sci Eng 63(1-4):18-22

Chan A, Kawaji M, Nakamura H, Kukita Y (1999) Experimental study of two-phase pump performance using a full size nuclear reactor pump. Nucl Eng Des 193(1-2):159-172

Chirkov DV, Ankudinova AS, Kryukov AE, Cherny SG, Skorospelov VA (2018) Multi-objective shape optimization of a hydraulic turbine runner using efficiency, strength and weight criteria. Struct Multidisc Optim 58(2):627-640

Choi Y-D, Kurokawa J, Imamura H (2007) Suppression of cavitation in inducers by J-Grooves. J Fluids Eng 129(1):15-22

d'Agostino L, Cervone A, Torre A, Pace G, Valentini D, Pasini A (2017) An introduction to flow-induced instabilities in rocket engine inducers and turbopumps. In: Cavitation Instabilities and Rotordynamic Effects in Turbopumps and Hydroturbines, Springer, Berlin, 65-86

Daróczy L, Janiga G, Thévenin D (2014) Systematic analysis of the heat exchanger arrangement problem using multi-objective genetic optimization. Energy 65:364-373

Daróczy L, Janiga G, Thévenin D (2016) Analysis of the performance of a H-Darrieus rotor under uncertainty using Polynomial Chaos Expansion. Energy 113:399-412

Daróczy L, Janiga G, Thévenin D (2018) Computational fluid dynamics based shape optimization of airfoil geometry for an H-rotor using a genetic algorithm. Eng Opt 50(9):1483-1499
Deb K, Pratap A, Agarwal S, Meyarivan T (2002) A fast and elitist multiobjective genetic algorithm: NSGA-II. IEEE Evolut Comput 6(2):182-197

Derakhshan S, Bashiri M (2018) Investigation of an efficient shape optimization procedure for centrifugal pump impeller using eagle strategy algorithm and ANN (case study: slurry flow). Struct Multidisc Optim 58(2):459-473

Edsall RM (2003) The parallel coordinate plot in action: design and use for geographic visualization. Comput Stat Data Anal 43(4):605-619

El Samanody M, Ghorab A, Mostafa MAF (2014) Investigations on the performance of centrifugal pumps in conjunction with inducers. Ain Shams Eng J 5(1):149-156

Fu Y, Yuan J, Yuan S, Pace G, d'Agostino L (2017) Effect of tip clearance on the internal flow and hydraulic performance of a threebladed inducer. Int J Rotating Mach 1-10

Furukawa A, Shirasu S, Sato S (1995) Experimental study of gasliquid two-phase flow pumping action of centrifugal impeller. ASME Publ FED 226:89-96

Gamboa J, Prado M (2011) Review of electrical-submersible-pump surging correlation and models. SPE Prod Oper 26(04):314-324

Gülich JF (2008) Centrifugal pumps. Springer, Berlin

Guo X, Zhu L, Zhu Z, Cui B, Li Y (2015) Numerical and experimental investigations on the cavitation characteristics of a highspeed centrifugal pump with a splitter-blade inducer. J Mech Sci Technol 29(1):259-267

Guo XM, Zhu ZC, Cui BL, Shi GP (2016) Effects of the number of inducer blades on the anti-cavitation characteristics and external performance of a centrifugal pump. J Mech Sci Technol 30(7):3173-3181

Han X, Kang Y, Sheng J, Hu Y, Zhao W (2020) Centrifugal pump impeller and volute shape optimization via combined NUMECA, genetic algorithm, and back propagation neural network. Struct Multidisc Optim 61(1):381-409

Hong S-S, Kim J-S, Choi C-H, Kim J (2006) Effect of tip clearance on the cavitation performance of a turbopump inducer. J Propuls Power 22(1): 174-179

Huang RF, Luo XW, Ji B, Wang P, Yu A, Zhai ZH, Zhou JJ (2015) Multi-objective optimization of a mixed-flow pump impeller using modified NSGA-II algorithm. Sci China 58(12):2122-2130

Hundshagen M, Mansour M Thévenin D Skoda R (2019) Numerical investigation of two-phase air-water flow in a centrifugal pump with closed or semi-open impeller. In: Proceedings of 13th European Turbomachinery Conference on Turbomachinery Fluid Dynamics and Thermodynamics, ETC 2019

Hundshagen M, Mansour M, Thévenin D, Skoda R (2021) 3D simulation of gas-laden liquid flows in centrifugal pumps and the assessment of two-fluid CFD methods. Exp Comput Multiphase Flow 3(3): 186-207

Inselberg A (2009) Parallel coordinates: visual multidimensional geometry and its applications. Springer, Berlin

Ji L, Li W, Shi W, Chang H, Yang Z (2020) Energy characteristics of mixed-flow pump under different tip clearances based on entropy production analysis. Energy 199:117447

Jiang Q, Heng Y, Liu X, Zhang W, Bois G, Si Q (2019) A review of design considerations of centrifugal pump capability for handling inlet gas-liquid two-phase flows. Energies 12(6):1078-1096

Kerikous E, Thévenin D (2019) Optimal shape of thick blades for a hydraulic Savonius turbine, Renew. Energy 134:629-638

Kim C, Kim S, Choi CH, Baek J (2017) Effects of inducer tip clearance on the performance and flow characteristics of a pump in a turbopump. Proc IMechE Part A 231(5):398-414

Kipouros T, Inselberg A, Parks G, Savill AM (2013) Parallel coordinates in computational engineering design. In: 54th AIAA/ASME/ 
ASCE/AHS/ASC Structures, Structural Dynamics, and Materials Conference, 1750

Kopparthy S, Mansour M, Janiga G, Thévenin D (2020) Numerical investigations of turbulent single-phase and two-phase flows in a diffuser. Int J Multiphase Flow 130:103333

Lundgreen R, Maynes D, Gorrell S, Oliphant K (2019) Increasing inducer stability and suction performance with a stability control device. J Fluids Eng 141(1):011204

Mansour M, Wunderlich B, Thévenin D (2018a) Experimental study of two-phase air/water flows in a centrifugal pump working with a closed or a semi-open impeller. In: ASME Turbo Expo 2018: Turbomachinery Technical Conference and Exposition, Oslo, Norway, 2018, V009T27A012

Mansour M, Wunderlich B, Thévenin D (2018b) Effect of tip clearance gap and inducer on the transport of two-phase air-water flows by centrifugal pumps. Exp Therm Fluid Sci 99:487-509

Mansour M, Kováts P, Wunderlich B, Thévenin D (2018c) Experimental investigations of a two-phase gas/liquid flow in a diverging horizontal channel. Exp Therm Fluid Sci 93:210-217

Mansour M, Parikh T, Engel S, Wunderlich B, Thévenin D (2019) Investigation on the influence of an inducer on the transport of single and two-phase air-flows by centrifugal pumps. In: 48th Turbomachinery \& 35th Pump Symposia

Mansour M, Parikh T, Engel S, Wunderlich B, Thévenin D (2020a) Numerical investigations of gas-liquid two-phase flow in a pump inducer. J Fluids Eng 142(2):021302

Mansour M, Parikh M, Thévenin D (2020b) Influence of blade pitch and number of blades of a pump inducer on single and two-phase flow performance. In: ASME Turbo Expo 2020: Turbomachinery Technical Conference and Exposition, Virtual Conference, 2020, V009T21A010

Mansour M, Zähringer K, Nigam KD, Thévenin D, Janiga G (2020c) Multi-objective optimization of liquid-liquid mixing in helical pipes using Genetic Algorithms coupled with Computational Fluid Dynamics. Chem Eng J 391:123570

Manzano Ruiz JJ (1980) Experimental and theoretical study of twophase flow in centrifugal pumps, Ph.D. thesis, Massachusetts Institute of Technology

Menter FR (1994) Two-equation eddy-viscosity turbulence models for engineering applications. AIAA J 32(8):1598-1605

Merry H (1976) Effects of two-phase liquid/gas flow on the performance of centrifugal pumps. In: Pumps compressors offshore oil gas, IMechE Conf. C130/76, 61

Monte Verde W, Biazussi JL, Sassim NA, Bannwart AC (2017) Experimental study of gas-liquid two-phase flow patterns within centrifugal pumps impellers. Exp Therm Fluid Sci 85:37-51

Murakami M, Minemura K (1974) Effects of entrained air on the performance of centrifugal pumps: 2nd report, effects of number of blades. Bull JSME 17(112):1286-1295

Nariman-Zadeh N, . Amanifard N, . Hajiloo N, Ghalandari P, Hoseinpoor B (2007) Multi-objective pareto optimization of centrifugal pump using genetic algorithms. In: Proceedings of the 11th WSEAS International Conference on COMPUTERS, 135-139

Neumann M, Schäfer T, Bieberle A, Hampel U (2016) An experimental study on the gas entrainment in horizontally and vertically installed centrifugal pumps. J Fluids Eng 138(9):91301

Nicholas PE, Padmanaban K, Vasudevan D, Ramachandran T (2015) Stacking sequence optimization of horizontal axis wind turbine blade using FEA, ANN and GA. Struct Multidisc Optim 52(4):791-801

Oshima M (1967) A study on suction performance of a centrifugal pump with an inducer. Bull JSME 10(42):959-965

Parikh T, Mansour M, Thévenin D (2020) Investigations on the effect of tip clearance gap and inducer on the transport of air-water twophase flow by centrifugal pumps. Chem Eng Sci 218:115554
Pouffary B, Patella RF, Reboud J-L, Lambert P-A (2008) Numerical analysis of cavitation instabilities in inducer blade cascade. $\mathbf{J}$ Fluids Eng 130(4):041302

Poullikkas A (2003) Effects of two-phase liquid-gas flow on the performance of nuclear reactor cooling pumps. Prog Nucl Energy 42(1):3-10

Pumps Sulzer (2013) Sulzer centrifugal pump handbook. Elsevier, New York, p 2013

Rodrigues SS, Marta AC (2020) Adjoint-based shape sensitivity of multi-row turbomachinery. Struct Multidisc Optim 61(2):837-853

Sá L, Novotny A, Romero J, Silva E (2017) Design optimization of laminar flow machine rotors based on the topological derivative concept. Struct Multidisc Optim 56(5):1013-1026

Sá L, Romero J, Horikawa O, Silva E (2018) Topology optimization applied to the development of small scale pump. Struct Multidisc Optim 57(5):2045-2059

Safikhani H, Khalkhali A, Farajpoor M (2011) Pareto based multiobjective optimization of centrifugal pumps using CFD, neural networks and genetic algorithms. Eng Appl Comput Fluid Mech $5(1): 37-48$

Sarkar S, Balakrishnan L(1990) Application of a Reynolds stress turbulence model to the compressible shear layer. In: 21st Fluid Dynamics, Plasma Dynamics and Lasers Conference, 1465

Sato S, Furukawa A, Takamatsu Y (1996) Air-water two-phase flow performance of centrifugal pump impellers with various blade angles. JSME Int J Ser B 39(2):223-229

Sauer M (2003) Einfluss der Zuströmung auf das Förderverhalten von Kreiselpumpen radialer Bauart bei Flüssigkeits-/Gasförderung, Ph.D. thesis, Technische Universität Kaiserslautern

Schäfer T, Neumann M, Bieberle A, Hampel U (2017) Experimental investigations on a common centrifugal pump operating under gas entrainment conditions. Nucl Eng Des 316:1-8

Schiavello B (1986) Two-phase flow rotodynamic pumps-experiments and design criteria. Pumps Offshore, Course, no. 7133, Worthing ton Simson Ltd

Shojaeefard MH, Hosseini SE, Zare J (2019) CFD simulation and Pareto-based multi-objective shape optimization of the centrifugal pump inducer applying GMDH neural network, modified NSGAII, and TOPSIS. Struct Multidisc Optim 60(4):1509-1525

Si Q, Bois G, Zhang K, Yuan J (2017) Air-water two-phase flow experimental and numerical analysis in a centrifugal pump. In: Proceedings of the 12th European Conference on Turbomachinery, Fluid Dynamics and Thermodynamics, Stockholm, Sweden, 2017, 3-7

Si Q, Bois G, Jiang Q, He W, Ali A, Yuan S (2018) Investigation on the handling ability of centrifugal pumps under air-water two-phase inflow: model and experimental validation. Energies 11(11):3048-3065

Siemens, STAR-CCM+ Version 13.02.013 User Guide (2018)

Song W, Keane A (2005) An efficient evolutionary optimisation framework applied to turbine blade firtree root local profiles. Struct Multidisc Optim 29(5):382-390

Song W-W, Wei L-C, Fu J, Shi J-W, Yang X-X, Xu Q-Y (2016) Analysis and control of flow at suction connection in high-speed centrifugal pump. Adv Mech Eng 9(1):1687814016685293

Speziale CG, Sarkar S, Gatski TB (1991) Modelling the pressurestrain correlation of turbulence: an invariant dynamical systems approach. J Fluid Mech 227:245-272

Stel H, Ofuchi EM, Sabino RH, Ancajima FC, Bertoldi D, Neto MAM, Morales RE (2019) Investigation of the motion of bubbles in a centrifugal pump impeller. J Fluids Eng 141(3):031203

Thévenin D, Janiga G (2008) Optimization and computational fluid dynamics. Springer, Berlin

Thum D (2007) Untersuchung von Homogenisierungseinrichtungen auf das Förderverhalten radialer Kreiselpumpen bei gasbeladenen Strömungen, Ph.D. thesis, Technische Universität Kaiserslautern 
Tillack P (1998) Förderverhalten von Kreiselpumpen bei viskosen, gasbeladenen Flüssigkeiten, Ph.D. thesis, Technische Universität Kaiserslautern

Zhang J, Zhu H, Yang C, Li Y, Wei H (2011) Multi-objective shape optimization of helico-axial multiphase pump impeller based on NSGA-II and ANN. Energy Convers Manag 52(1):538-546

Zhu Z, Xie P, Ou G, Cui B, Li Y (2008) Design and experimental analyses of small-flow high-head centrifugal-vortex pump for gas-liquid two-phase mixture. Chin J Chem Eng 16(4):528-534
Zhu J, Guo X, Liang F, Zhang HQ (2017) Experimental study and mechanistic modeling of pressure surging in electrical submersible pump. J Nat Gas Sci Eng 45:625-636

Publisher's Note Springer Nature remains neutral with regard to jurisdictional claims in published maps and institutional affiliations. 